\title{
Six years of BeppoSAX observations of blazars: A spectral catalog ${ }^{\star}$
}

\author{
D. Donato ${ }^{1}$, R. M. Sambruna ${ }^{1,2}$, and M. Gliozzi ${ }^{1,2}$ \\ ${ }^{1}$ George Mason University, School of Computational Sciences, 4400 University Drive, Fairfax, VA 22030, USA \\ e-mail: davide@physics.gmu.edu \\ 2 George Mason University, Dept. Of Physics \& Astronomy, MS 3F3, 4400 University Drive, Fairfax, VA 22030, USA
}

Received 21 October 2003 / Accepted 13 December 2004

\begin{abstract}
We present a spectral catalog for blazars based on the BeppoSAX archive. The sample includes 44 High-energy peaked BL Lacs (HBLs), 14 Low-energy peaked BL Lacs (LBLs), and 28 Flat Spectrum Radio Quasars (FSRQs). A total of 168 LECS, MECS, and PDS spectra were analyzed, corresponding to observations taken in the period 1996-2002. The $0.1-50 \mathrm{keV}$ continuum of LBLs and FSRQs is generally fitted by a single power law with Galactic column density. A minority of the observations of LBLs (25\%) and FSRQs (15\%) is best fitted by more complex models like the broken power law or the continuously curved parabola. These latter models provide also the best description for half of the HBL spectra. Complex models are more frequently required for sources with fluxes $F_{2-10 \mathrm{keV}}>10^{-11} \mathrm{erg} \mathrm{cm}^{-2} \mathrm{~s}^{-1}$, corresponding to spectra with higher signal-to-noise ratio. As a result, considering sources with flux above this threshold, the percentage of spectra requiring those models increases for all the classes. We note that there is a net separation of X-ray spectral properties between HBLs on one side, and LBLs and FSRQs on the other, the distinction between LBLs and FSRQs is more blurry. This is most likely related to ambiguities in the optical classification of the two classes.
\end{abstract}

Key words. galaxies: active - galaxies: fundamental parameters - galaxies: nuclei - X-rays: galaxies - catalogs

\section{Introduction}

Blazars are a class of radio-loud Active Galactic Nuclei (AGN) defined by non-thermal emission from a jet oriented close to the line of sight. Multiwavelength blazar studies have made it clear that their spectral energy distributions (SEDs) are characterized, in a $v-v F(v)$ plot, by two broad peaks (Giommi \& Padovani 1994). The first component, peaking anywhere in the IR-soft X-ray band, is due to synchrotron emission, while the higher-energy one is due to the inverse Compton emission of ambient photons off the same electrons producing the synchrotron part of the spectrum (Maraschi et al. 1992; Sikora et al. 1994; but see Mannheim 1993 for a different interpretation).

Previous studies have shown that low-luminosity BL Lacs (High-energy peaked BL Lacs, or HBLs) exhibit the synchrotron peak in the UV-soft X-ray band, and the inverse Compton peak between the $\mathrm{GeV}$ and the $\mathrm{TeV}$ band (Sambruna et al. 1994; Giommi et al. 1995; Padovani \& Giommi 1995; Fossati et al. 1997). The two components have approximately the same power. For mid-luminosity sources (Low-energy peaked BL Lacs, or LBLs) the synchrotron peak is in the near infrared band and the X-ray emission is due either to the

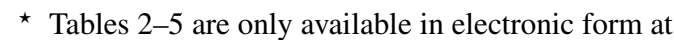
http://www . edpsciences.org synchrotron or the Compton component, or both. For the highluminosity sources (Flat Spectrum Radio Quasars, or FSRQs) the synchrotron peak is in the far infrared band while X-ray emission is ascribed to the Compton component.

The X-ray band, at the overlap of the synchrotron and Compton components, is key to disentangle the contribution of the two components to the broad band continuum. Based on current unification studies (e.g., Fossati et al. 1997) one expects the low-luminosity sources to be dominated by the high-energy tail of the synchrotron emission in the X-rays, and thus to have steep (photon index $\Gamma>2$, where $F_{v} \propto v^{-(\Gamma-1)}$ ) or convex $\mathrm{X}$-ray spectra, as a result of radiative losses. On the other hands, FSRQs should have flatter spectra $(\Gamma<2)$, while LBLs should exhibit intermediate slopes, or even concave X-ray continua.

Previous systematic analysis of the X-ray continua of blazars relied on satellites with limited sensitivity (e.g., EXOSAT; Sambruna et al. 1994a,b) or limited bandpass (ROSAT, Urry et al. 1996; Perlman et al. 1996; ASCA, Sambruna et al. 1999; Donato et al. 2001). These studies showed that the X-ray spectra of blazars are often complex, with downward-curved continua in HBLs and occasionally upward-curved continua in FSRQs and LBLs.

With its wide X-ray band pass (0.1-200 keV) and good sensitivity, BeppoSAX is the instrument of choice to study the broadband X-ray continua of blazars. The BeppoSAX mission 
ended in early 2002; during its lifetime, BeppoSAX observed a large number of blazars as part of multiwavelength campaigns or to obtain snapshot X-ray spectra. The BeppoSAX observations for individual sources were published by the original PIs of the investigation. Here we present a uniform analysis of all the public LECS, MECS, and PDS observations, focusing on the spectral properties. A similar previous account was given by Giommi et al. (2002).

The paper is organized as follows. In Sect. 2 we describe the sample selection, and in Sect. 3 the data reduction. In Sect. 4 the spectral catalog is described, while in Sect. 5 we discuss the results. Throughout this paper, $H_{0}=75 \mathrm{~km} \mathrm{~s}^{-1} \mathrm{Mpc}^{-1}$ and $q_{0}=0.5$ are adopted.

\section{Sample selection}

The BeppoSAX satellite carried onboard four Narrow Field Instruments (NFI) pointing in the same direction and covering a very large energy range from 0.1 to $300 \mathrm{keV}$ (Boella et al. 1997). Two of the four instruments have imaging capability, the Low Energy Concentrator Spectrometer (LECS), sensitive in the nominal range $0.1-10 \mathrm{keV}$, and three Medium Energy Concentrator Spectrometers (MECS), sensitive in the nominal range $1-10 \mathrm{keV}$. The other two detectors are the High Pressure Proportional Counter (HPGSPC), sensitive in the nominal range 4-120 keV, and the Phoswich Detector System (PDS), sensitive in the nominal range $13-300 \mathrm{keV}$. We restricted our analysis to the LECS, MECS, and PDS archives, as the HPGSPC has not enough sensitivity to detect relatively faint sources as blazars.

We cross-correlated the BeppoSAX database with published lists of blazars, including Fossati et al. (1997), Donato et al. (2001), and Giommi et al. (2002), and references herein. Following Padovani \& Giommi (1995), sources with the radioto-X-ray spectral index, $\alpha_{\mathrm{rx}}$, smaller than 0.75 were classified as HBLs, while sources with $\alpha_{\mathrm{rx}} \gtrsim 0.75$ were classified as LBLs or FSRQs, depending on the EW of the optical emission lines.

The sample is presented in Table 1, where we list the name of the object (Col. 1), the coordinates for the equinox 2000.0 (Cols. 2 and 3), the redshift (Col. 4), the Galactic column density $N_{\mathrm{H}}^{\mathrm{Gal}}$ (Col. 5), and the number of BeppoSAX observations of the source (Col. 6). The Galactic column was derived from Dickey \& Lockman (1990). The sample includes 86 blazars: 44 HBLs, 14 LBLs, and 28 FSRQs. Most sources were observed as part of multiwavelength campaigns, or in a single snapshot observation to obtain the Spectral Energy Distributions. Since the BeppoSAX selection process favored objects known for their high X-ray brightness, the sample is highly heterogeneous (with some sources observed multiple times), strongly biased, and by no means complete.

\section{Observations and data analysis}

All public observations up to January 2002 were analyzed, for a total of 168 spectra. In Table 2 we present the log of the observations, together with the exposure times and the mean count rates in the various instruments. For each observation we report
Table 1. The sample.

\begin{tabular}{|c|c|c|c|c|c|}
\hline $\begin{array}{c}\text { Obj. name } \\
\text { (1) }\end{array}$ & $\begin{array}{c}\mathrm{RA}(\mathrm{J} 2000) \\
(2)\end{array}$ & $\begin{array}{c}\operatorname{Dec}(\mathrm{J} 2000) \\
\text { (3) }\end{array}$ & $\begin{array}{c}\text { Redshift } \\
\text { (4) }\end{array}$ & $\begin{array}{c}N_{\mathrm{H}, \mathrm{Gal}} \\
10^{20} \\
(5)\end{array}$ & $\begin{array}{c}\text { No. of } \\
\text { observ. } \\
(6)\end{array}$ \\
\hline \multicolumn{6}{|c|}{ HBLs } \\
\hline $1 \mathrm{ES} 0033+595$ & 003552.5 & +595003.9 & 0.086 & 42.7 & \\
\hline $1 \mathrm{ES} 0120+340$ & 012308.5 & +342048.9 & 0.272 & 5.14 & 2 \\
\hline RX J0136.5+3905 & 013632.8 & +390556.0 & - & 6.01 & \\
\hline 1ES $0145+138$ & 014829.8 & +140216.0 & 0.125 & 5.18 & \\
\hline MS $0158.5+0019$ & 020106.2 & +003400.0 & 0.298 & 2.67 & \\
\hline $1 \mathrm{ES} 0229+200$ & 023248.4 & +201716.0 & 0.140 & 9.32 & \\
\hline MS $0317.0+1834$ & 031951.8 & +184535.0 & 0.190 & 10.4 & \\
\hline $1 \mathrm{ES} 0323+022$ & 032613.9 & +022514.9 & 0.147 & 8.81 & \\
\hline 1ES 0347-121 & 034923.2 & -115927.0 & 0.185 & 3.63 & \\
\hline 1ES $0414+009$ & 041652.4 & +010524.0 & 0.287 & 10.5 & \\
\hline 1ES $0502+675$ & 050756.2 & +673724.0 & 0.314 & 9.11 & \\
\hline 1ES 0507-040 & 050938.2 & -040046.0 & 0.304 & 9.74 & \\
\hline PKS 0548-322 & 055040.6 & -321614.9 & 0.069 & 2.20 & 3 \\
\hline MS 0737.9+7441 & 074405.2 & +743358.0 & 0.315 & 3.29 & \\
\hline В20912+29 & 091552.3 & +293320.0 & - & 1.97 & \\
\hline $1 \mathrm{ES} 0927+500$ & 093037.6 & +495026.9 & 0.188 & 1.40 & \\
\hline $1 \mathrm{ES} 1028+511$ & 103118.4 & +505336.0 & 0.361 & 1.15 & \\
\hline RX J1037.7+5711 & 103744.2 & +571154.9 & - & 0.55 & \\
\hline RX J1058.6+5628 & 105837.6 & +562811.9 & 0.144 & 0.68 & 2 \\
\hline 1ES 1101-232 & 110337.6 & -232930.9 & 0.186 & 6.05 & 2 \\
\hline Mkn 421 & 110427.3 & +381232.0 & 0.030 & 1.34 & 16 \\
\hline RX J1117.1+2014 & 111706.1 & +201407.0 & 0.139 & 1.33 & \\
\hline 1ES $1118+424$ & 112048.0 & +421212.0 & 0.124 & 1.93 & \\
\hline 1ES $1133+704$ & 113626.4 & +700928.0 & 0.045 & 1.40 & \\
\hline RX J1211.9+2242 & 121158.0 & +224236.0 & 0.455 & 2.22 & 3 \\
\hline ON325 & 121752.0 & +300700.9 & 0.130 & 1.70 & 2 \\
\hline 1ES $1218+304$ & 122121.7 & +301036.9 & 0.182 & 1.71 & \\
\hline $1 \mathrm{ES} 1255+244$ & 125731.9 & +241238.9 & 0.141 & 1.34 & \\
\hline MS 1312.1-4221 & 131503.7 & -423650.0 & 0.108 & 8.45 & \\
\hline 1 RXS J141756.8+25 & 141756.7 & +254328.9 & 0.237 & 1.58 & 3 \\
\hline $1 \mathrm{ES} 1426+428$ & 142832.5 & +424018.9 & 0.129 & 1.36 & \\
\hline MS $14588+2249$ & 150102.8 & +223754.9 & 0.235 & 3.54 & \\
\hline $1 \mathrm{ES} 1517+656$ & 151747.6 & +652523.9 & 0.702 & 1.93 & \\
\hline 1ES $1533+535$ & 153500.7 & +532038.0 & 0.890 & 1.32 & \\
\hline 1ES $1544+820$ & 154015.5 & +815504.0 & - & 4.32 & \\
\hline 1ES $1553+113$ & 155543.1 & +111120.0 & 0.360 & 3.67 & \\
\hline Mkn 501 & 165352.2 & +394537.0 & 0.034 & 1.81 & 11 \\
\hline $\mathrm{H} 1722+119$ & 172504.3 & +115215.9 & 0.018 & 8.86 & \\
\hline 1ES $1741+196$ & 174357.7 & +193508.9 & 0.084 & 6.68 & \\
\hline 1ES $1959+650$ & 195959.8 & +650854.9 & 0.047 & 9.89 & 3 \\
\hline PKS 2005-489 & 200925.3 & -484953.0 & 0.071 & 5.09 & 2 \\
\hline PKS 2155-304 & 215851.7 & -301328.9 & 0.116 & 1.70 & 3 \\
\hline $1 \mathrm{ES} 2344+514$ & 234704.8 & +514217.9 & 0.044 & 15.4 & 7 \\
\hline H2356-309 & 235907.6 & -303736.9 & 0.165 & 1.37 & \\
\hline \multicolumn{6}{|c|}{ LBLs } \\
\hline PKS 0048-09 & 005041.2 & -092905.9 & - & 3.85 & \\
\hline $3 \mathrm{C} 66 \mathrm{~A}$ & 022239.6 & +430208.0 & 0.444 & 9.41 & 2 \\
\hline $\mathrm{AO} 0235+164$ & 023838.8 & +163659.0 & 0.940 & 8.85 & \\
\hline PKS 0537-441 & 053850.3 & -440508.9 & 0.894 & 3.94 & \\
\hline S50716+71 & 072153.4 & +712035.9 & - & 3.84 & 3 \\
\hline $\mathrm{OJ} 287$ & 085449.0 & +200632.0 & 0.306 & 3.05 & 2 \\
\hline PKS 1144-379 & 114701.4 & -381211.0 & 1.048 & 7.53 & \\
\hline ON231 & 122131.6 & +281358.0 & 0.102 & 1.88 & 2 \\
\hline OQ530 & 141946.6 & +542316.0 & 0.151 & 1.18 & 3 \\
\hline PKS 1519-273 & 152237.7 & -273011.0 & 0.071 & 8.41 & \\
\hline S51803+784 & 180045.7 & +782804.0 & 0.680 & $\begin{array}{l}0.41 \\
3.94\end{array}$ & \\
\hline 3 C 371 & 180650.6 & $\begin{array}{r}094927.9 \\
+69\end{array}$ & 0.051 & 4.74 & \\
\hline $4 C 56.27$ & 182407.1 & +565100.0 & 0.664 & 4.14 & \\
\hline BLLAC & 220243.3 & +421640.0 & 0.069 & 20.1 & 5 \\
\hline \multicolumn{6}{|c|}{ FSRQs } \\
\hline PKS 0208-512 & 021046.1 & -510101.9 & 0.999 & 3.09 & \\
\hline NRAO140 & 033629.8 & +321820.0 & 1.258 & 15 & \\
\hline PKS 0521-365 & 052258.0 & -362730.9 & 0.055 & 3.45 & \\
\hline PMN 0525-3343 & 052506.2 & -334305.0 & 4.401 & 2.24 & \\
\hline PKS $0528+134$ & 053056.4 & +133155.0 & 2.060 & 25.7 & 8 \\
\hline WGAJ0546.6-6415 & 054641.8 & -641521.9 & 0.323 & 4.44 & \\
\hline PKS 0743-006 & 074554.1 & -004417.0 & 0.994 & 6.48 & \\
\hline $1 \mathrm{ES} 0836+710$ & 084124.4 & +705341.9 & 2.172 & 2.78 & \\
\hline RGB J0909+039 & 090915.8 & +035442.1 & 3.200 & 3.48 & \\
\hline PKS $1127-14$ & 113007.0 & -144927.1 & 1.187 & 3.83 & \\
\hline 3 C 273 & 122906.7 & +020309.0 & 0.158 & 1.79 & 9 \\
\hline $3 \mathrm{C} 279$ & 125611.1 & -054722.0 & 0.536 & 2.26 & 5 \\
\hline GB $1428+4217$ & 143023.8 & +420436.9 & 4.715 & 1.38 & \\
\hline GB $1508+5714$ & 151002.8 & +570244.9 & 4.301 & 1.40 & 2 \\
\hline PKS 1510-089 & 151250.4 & -090559.9 & 0.360 & 8.18 & \\
\hline
\end{tabular}


Table 1. continued.

\begin{tabular}{lccccc}
\hline \multicolumn{1}{c}{ Obj. name } & RA(J2000) & Dec(J2000) & Redshift & $\begin{array}{c}N_{\mathrm{H}, \mathrm{Gal}} \\
10^{20} \mathrm{~cm}^{-2}\end{array}$ & $\begin{array}{c}\text { No. of } \\
\text { observ. } \\
(1)\end{array}$ \\
\hline RGB J1629+4008 & 162901.3 & +400758.0 & 0.272 & 0.84 & \\
1ES 1641+399 & 164258.8 & +394837.0 & 0.593 & 1.01 & \\
RGB J1722+2436 & 172241.3 & +243619.0 & 0.175 & 4.71 & \\
S41745+62 & 174613.9 & +622656.0 & 3.889 & 3.43 & 2 \\
S5 2116+81 & 211401.2 & +820447.9 & 0.084 & 7.60 & 2 \\
PKS 2126-158 & 212912.1 & -153841.9 & 3.268 & 4.92 & \\
PKS 2134+004 & 213638.5 & +004153.9 & 1.932 & 4.48 & \\
PKS 2149-306 & 215155.3 & -302753.9 & 2.345 & 2.11 & \\
PKS 2223+210 & 222537.9 & +211806.9 & 1.959 & 4.37 & \\
PKS 2223-05 & 222547.2 & -045702.9 & 1.404 & 5.65 & \\
H2230+114 & 223236.4 & +114350.9 & 1.037 & 5.00 & 5 \\
PKS 2243-123 & 224618.1 & -120650.0 & 0.630 & 4.78 & \\
H2251+158 & 225357.7 & +160853.9 & 0.859 & 6.55 & \\
\hline
\end{tabular}

Columns: 1 = object name as found in BeppoSAX archive; $2=$ Right Ascension (at J2000); 3 = Declination (at J2000); 4 = redshift; 5 = Galactic absorption based on Dickey \& Lockman (1990); 6 = number of observations in the BeppoSAX archive.

the LECS count rates in the energy range $0.1-2 \mathrm{keV}$ and the MECS count rates in the energy range 2-10 keV. Inspection of the PDS spectra shows that the background usually dominates above $50 \mathrm{keV}$. Thus, counts were extracted in the energy range $13-50 \mathrm{keV}$ for all sources. However, for the brightest sources reliable counts up to $200 \mathrm{keV}$ were detected. In these cases, spectral fits to the PDS data were performed in the energy range $0.1-200 \mathrm{keV}$.

In Table 2, we report the PDS count rates for only those sources which were detected at $\gtrsim 3 \sigma$ confidence level. Most of the sources are weak at energies greater than $10 \mathrm{keV}$ and only 36 sources are detected (15 HBLs, 4 LBLs, and 17 FSRQs).

We extracted LECS, MECS, and PDS spectra. The data analysis for the LECS and MECS instruments was based on the linearized, cleaned event files obtained from the on-line archive. The spectra were extracted with the FTOOLS package XSELECT (v. 2.2), using extraction regions of radius of $8^{\prime}$ and $4^{\prime}$ for the LECS and MECS, respectively. In the case of weak sources, we used a radius of $6^{\prime}$ and sometimes even $4^{\prime}$ to extract the LECS spectra. The LECS and MECS background is low but not uniformly distributed across the detector. For this reason it is better to evaluate the background from blank fields that are available from the SDC public ftp site. Since these background data were taken in regions of the sky with no detected sources and low Galactic absorption, the low energy $\mathrm{X}$-ray counts coming from sources located in regions with high Galactic absorption may be underestimated and/or the intrinsic absorption may be overestimated.

The spectral analysis was performed with XSPEC v.11.2, using the latest available response matrices from the BeppoSAX calibration center. The spectra from LECS, MECS, and PDS detectors were rebinned using the channel grouping suggested by the BeppoSAX team which was designed to match the detector resolution and sensitivity in an optimal way. The LECS/MECS and MECS/PDS normalization factors (to account for inter-calibration systematics of the instruments) were left free to vary; normal acceptable values are in the range $0.65-1.0$ and $0.77-0.93$, respectively (Fiore et al. 1999). Spectral fits were performed in the energy ranges $0.1-2 \mathrm{keV}$ for the LECS, 2-10 keV for the MECS, and 13-200 keV for the PDS where the calibration is best known and the background contribution negligible. The best-fit models were determined using the $\chi^{2}$ minimization routine. The significance of the fit improvement, when additional free parameters were added, was evaluated using the F-test, assuming as a threshold for significant improvement $P_{\mathrm{F}}=95 \%$. Uncertainties on the fitted parameters are $90 \%$ confidence $\left(\Delta \chi^{2}=2.7\right)$ for one parameter of interest.

The following spectral models were used to fit the BeppoSAX spectra:

(1) a single power law with photon index $\Gamma$ and column density $N_{\mathrm{H}}$ fixed to the Galactic value;

(2) a single power law with free $N_{\mathrm{H}}$;

(3) a broken power law with break energy $E_{\text {break }}$, and photon indices below and above the break $\Gamma_{1}$ and $\Gamma_{2}$, respectively;

(4) a continuously curved parabola (Fossati et al. 2000). The latter has the same parameters as model 3), but $\Gamma_{1}$ and $\Gamma_{2}$ are evaluated at $1 \mathrm{keV}$ and $10 \mathrm{keV}$, respectively.

We used the following procedure. At first, all the spectra were fitted with model (1). When the fit was unsatisfactory, model (2) was used. In several cases, the fitted column density from model (2) was consistent with the Galactic value within the errors. We interpret this result as marginal evidence for continuum curvature; however, in these cases fits with the curved models (3) and (4) did not provide a significant improvement. The observations for which models (1) and (2) provided the best-fit are reported in Table 3 .

However, in the case of bright sources, $F_{2-10 \mathrm{keV}}>$ $10^{-11} \mathrm{erg} \mathrm{cm}^{-2} \mathrm{~s}^{-1}$, corresponding to spectra with higher signal-to-noise ratio, a curved model was generally found to provide a better description of the BeppoSAX spectra than either model (1) or (2). In few cases (6 observations) where the curved model with Galactic absorption did not provide a good description of the spectrum, the absorption was left free to vary. These observations are listed in Table 4. In Table 5 we also list the fits with models (1) and (2) for the same spectra, for future use. As a summary, the last column of Table 2 reports the model that best-fits the individual spectra for each source in the sample.

The choice of the above spectral models is motivated by previous X-ray studies of blazars (e.g., EXOSAT, Ginga, ASCA; Sambruna et al. 1994a; Tashiro et al. 1995; Takahashi et al. 1996; Donato et al. 2001). These authors found that the X-ray continua of high-luminosity blazars are usually well described by a single power law, while at decreasing luminosities curved $\mathrm{X}$-ray continua are often observed. The latter are convex, with $\Gamma_{1}<\Gamma_{2}$, in HBLs and concave, with $\Gamma_{1}>\Gamma_{2}$, in some LBLs and FSRQs. In the brightest HBLs, the broken power law model is inadequate to represent the continuous curvature of the X-ray spectra, and better results are obtained using a continuously curved model (i.e., Fossati et al. 2000 for MKN 421, Tavecchio et al. 2001 for MKN 501). 
An independent analysis of the BeppoSAX blazar archive was performed by Giommi et al. (2002), who analyzed a total of 157 X-ray spectra of 84 sources (42 HBLs, 12 LBLs, 22 FSRQs, and 4 GigaHertz Peaked Spectrum QSOs). These authors fitted the LECS+MECS+PDS spectra using a single power law, a broken power law, a sum of two power laws, and a logarithmic parabola. For each model the $N_{\mathrm{H}}$ was fixed at the Galactic value. Comparing the results for the models that are in common with this work (single power law or broken power law), we find that our results are completely consistent with Giommi et al. (2002).

\section{The spectral catalog}

The results of our spectral analysis, from fits to the joint LECS+MECS+PDS datasets in the energy range $0.1-200 \mathrm{keV}$, are reported in Tables 3 and 4. For each source we present the parameters obtained with the model that best-fits the data (power-law model in Table 3 and curved model in Table 4), and their $90 \%$ confidence uncertainties. For sources with multiple observations, different models best-fit the data at different epochs. We reported the individual best-fit models, using a different procedure than Giommi et al. 2002, who systematically used the same model for repeatedly observed sources.

For the HBL class we obtained spectral information for 90 observations of 44 sources. For 46 observations (corresponding to 15 sources) the model that best-fits the LECS+MECS+PDS data is a downward-curved model (broken power law or curved parabola), while the remaining observations are described by a single power law. In the latter cases, the photon index is steep, $\Gamma \gtrsim 2$. The energy break, in the cases of a curved model fit, is more frequently located around $1-2 \mathrm{keV}$. Although for most of the sources the best fit is obtained with intrinsic absorption fixed to the Galactic value, 20 spectral fits corresponding to 17 sources require a column density of the order of $10^{20} \mathrm{~cm}^{-2}$. This may indicate residual curvature in the continuum which the model is still inadequate to describe.

For the LBL class we analyzed 25 observations of 14 sources. For 7 observations (corresponding to 4 sources) the model that best-fits the LECS+MECS+PDS data is a curved model, while the remaining observations are described by a single power law. The photon indices of the single power laws are flat, $\Gamma<2$, in 9 sources (13 observations) and steep, $\Gamma \geq 2$, in 4 sources (4 observations). For the sources with a spectrum described by a curved model, S5 0716+71 and ON 231 need for all their observations an upward curvature, with a steep photon index below the break $\left(\Gamma_{1} \geq 2.4\right)$ and a flatter index above the break $\left(\Gamma_{2} \leq 2\right)$. One observation of $3 \mathrm{C} 66 \mathrm{~A}$ and one of BL Lac need a downward-curved model. In both sources, $\Gamma_{1} \sim 2.2$, while $\Gamma_{2} \sim 2.3$ and 2.6, respectively, while the break energy is at $0.3-0.4 \mathrm{keV}$. Among the sources with multiple observations, only BL Lac shows spectral variability, with a total change of the photon index $\Delta \Gamma \sim 1$ for a change of the flux of a factor 3 (from 6 to $20 \times 10^{-12} \mathrm{erg} \mathrm{cm}^{-2} \mathrm{~s}^{-1}$ ).

Finally, for the FSRQ class 53 observations (corresponding to 28 sources) were analyzed. The best-fit is always obtained using a single power law (model (1)) with $\Gamma \leq 1.8$, except for
1ES 0836+710, PKS 1510-089, PKS 2126-158, and 5 observations of $3 \mathrm{C} 273$, for which a curved model is needed. All the observations are fitted using the intrinsic absorption fixed to the Galactic value. For 1ES 0836+710 and PKS 2126-158 downward curvature is detected, with $\Gamma_{1} \sim 1$ and $\Gamma_{2} \sim 1.3$ and 1.8, respectively. For 3C 273 and PKS 1510-089 upward curvature is indicated, indicating the presence of a soft excess.

Since the soft excess can have thermal origins (e.g., accretion disk), we fitted the BeppoSAX data with a power law plus either a blackbody or a bremsstrahlung. The inclusion of either thermal component was not statistically preferred to a broken power law. Thus, the origin of the soft excess in 3C 273 and PKS 1510-089 is still an open question.

\section{Results and discussion}

We presented a uniform analysis of the BeppoSAX archival spectra for blazars. We will now use the spectral catalog to infer the average X-ray properties of the three blazar classes. While these properties were investigated before with ASCA (Sambruna et al. 1999; Donato et al. 2001), only BeppoSAX has the unique combination of wide bandpass and sensitivity necessary to study the entire X-ray range. An important caveat, however, is that the "sample" is incomplete and by all means biased toward the brightest X-ray sources of each class.

First, we derive average X-ray spectral parameters for each best-fit model and each class of blazars. To avoid a bias toward sources observed multiple times, we adopted the following procedure. For sources observed only a few times $(\leq 9)$ and with negligible variations in spectral index $(\Delta \Gamma \lesssim 0.5)$ or flux variation of a factor of $\sim 2$ between maximum and minimum, we used the observation for which the spectral parameters are better constrained. For sources observed multiple times and with significant spectral or flux variations, we considered two observations, corresponding to the two most extreme values of the spectral index and/or flux. This procedure yielded 47 observations of $44 \mathrm{HBLs}$ (Mkn 421, Mkn 501, and 1ES 2344+514 were observed in 2 states), 15 observations of 14 LBLs (BL Lac showed variability both in the flux and in the spectral index), and 29 observations of the 28 FSRQs (PKS 0528+134 showed variability in the spectral index). The average parameters are reported in Table 6.

Second, in order to compare in a homogeneous way all the objects, we parameterize the continuum in $0.1-200 \mathrm{keV}$ with a simple power law plus fixed and free absorption, even for sources whose spectrum was better described by a curved model. Thus, we used the values of the photon index and luminosity from the fits reported in Tables 3 and 5. Using this approach, a convex/concave continuum will be represented by a power law with larger/smaller absorption column than Galactic. To reduce the bias toward sources with multiple observations, we adopted the procedure described above which reduces the number of observations to a maximum of 2 for the most variable sources.

The distributions of the spectral indices from the fits with a single power law model are shown in Fig. 1 for the three classes. Figure 2 shows the distributions for the monochromatic luminosities at $1 \mathrm{keV}$. The average values are reported in 


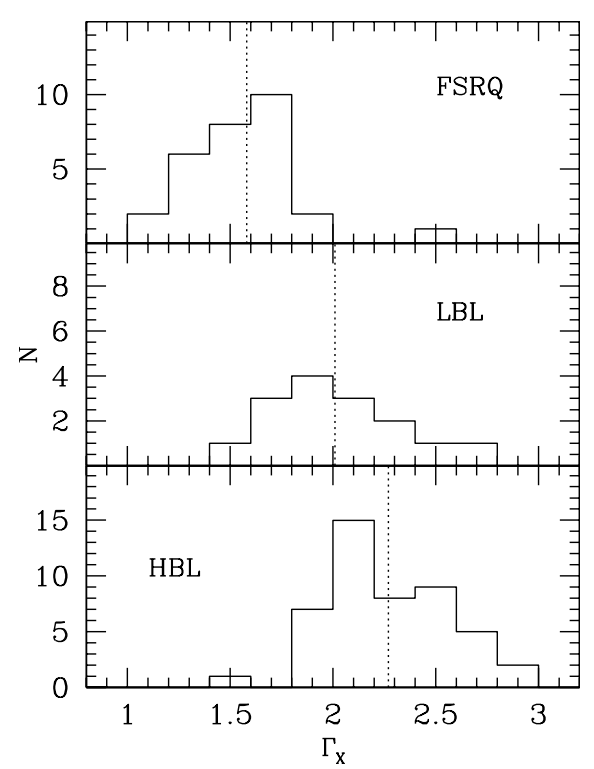

Fig. 1. Distribution of the photon spectral indices obtained using a single power law fit. The dotted line represent the average value reported in Table 7.

Table 6. Average spectral parameters for the best-fit models.

\begin{tabular}{l|ccc}
\hline \hline & HBL & LBL & FSRQ \\
\hline & \multicolumn{3}{|c}{ a) Single power law } \\
$N$ & 34 & 12 & 26 \\
$\left\langle\Gamma_{\mathrm{PL}}\right\rangle$ & $2.24(0.04)$ & $1.92(0.07)$ & $1.59(0.05)$ \\
\hline \multicolumn{4}{|c}{ b) Broken power law } \\
$N$ & 6 & 2 & 2 \\
$\left\langle\Gamma_{1}\right\rangle$ & $2.06(0.18)$ & $2.29(0.07)$ & $1.06(0.03)$ \\
$\left\langle E_{\text {break }}\right\rangle$ & $2.24(1.02)$ & $1.10(0.65)$ & $0.49(0.11)$ \\
$\left\langle\Gamma_{2}\right\rangle$ & $2.65(0.26)$ & $2.24(0.40)$ & $1.56(0.22)$ \\
\hline \multicolumn{4}{|c}{ c) Continuously curved parabola } \\
$N$ & 7 & 1 & 1 \\
$\left\langle\Gamma_{1}\right\rangle$ & $1.91(0.14)$ & 2.58 & 2.51 \\
$\left\langle E_{\text {break }}\right\rangle$ & $1.71(0.36)$ & 3.10 & 1.40 \\
$\left\langle\Gamma_{2}\right\rangle$ & $2.53(0.12)$ & 1.58 & 1.32 \\
\hline
\end{tabular}

The break energy is in $\mathrm{keV}$. For each value we report also the standard deviation (in parenthesis) and the number $N$ of observations used.

Table 7. To compare the distributions of the 3 classes in Figs. 1 and 2, we used a Kolmogorov-Smirnov test, which gives the probability $P_{\mathrm{KS}}$ that 2 distributions are drawn from the same parent population. Therefore, small values of $P_{\mathrm{KS}}$ indicate that the cumulative distribution function of the first data set is significantly different from that of the second data set. For the spectral index distributions in Fig. 1, $P_{\mathrm{KS}} \sim 0.23$ for HBLs vs. LBLs, $P_{\mathrm{KS}} \sim 0.99$ for HBLs vs. FSRQs, and $P_{\mathrm{KS}} \sim 0.83$ for LBLs vs. FSRQs. Thus, we conclude that there is no significant difference in the spectral index distribution among the three classes. However, the Kolmogorov-Smirnov test is sensitive only to the shape of the distribution, and not to the location of its centroid. We thus compared the average values of the

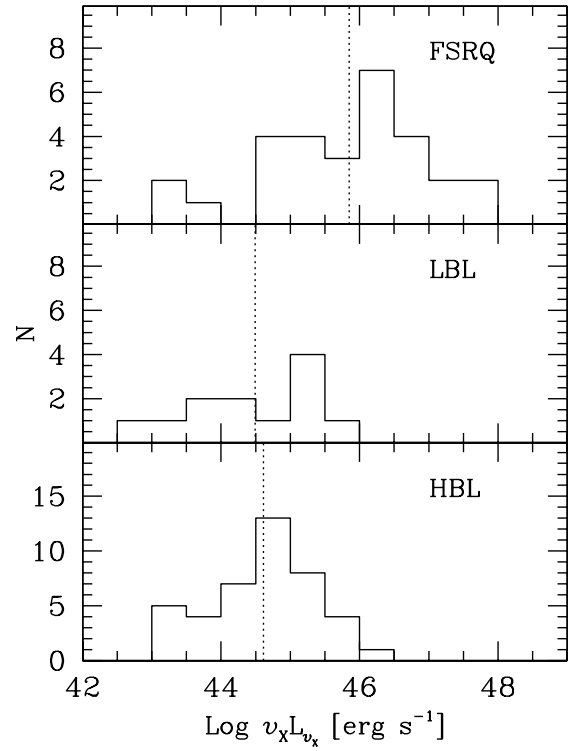

Fig. 2. Distribution of the $v L v$ monochromatic $(1 \mathrm{keV})$ luminosity for the three subclasses obtained using a single power law fit. The dotted line is the average spectral index from Table 7.

Table 7. Average parameters for the blazar classes.

\begin{tabular}{l|ccc}
\hline \hline & HBL & LBL & FSRQ \\
\hline$N$ & 47 & 15 & 29 \\
$\langle\Gamma\rangle$ & $2.27(0.04)$ & $2.01(0.08)$ & $1.58(0.05)$ \\
$\left\langle L_{\mathrm{X}}\right\rangle$ & $44.61(0.11)$ & $44.49(0.27)$ & $45.85(0.22)$ \\
\hline
\end{tabular}

Average values of the X-ray photon indices and monochromatic $1 \mathrm{keV}$ luminosities from fitting all the BeppoSAX spectra with either model (1) or model (2). The standard deviation are in parenthesis.

spectral index for the 3 subclasses in Table 7. Comparing the average indices for HBLs and LBLs and for LBLs and FSRQs we found that they are marginally consistent at $2 \sigma$ and $3 \sigma$, respectively. On the other hand, the average spectral index of HBLs is significantly different from the FSRQs, with HBLs having a steeper X-ray continua than FSRQs.

Similar conclusions are derived if the average luminosities of the three classes are compared. Using the KolmogorovSmirnov test, we find $P_{\mathrm{KS}} \sim 0.31$ for HBLs vs. LBLs, $P_{\mathrm{KS}} \sim 0.89$ for HBLs vs. FSRQs, and $P_{\mathrm{KS}} \sim 0.31$ for LBLs vs. FSRQs, suggesting that the distribution shapes are drawn from the same population. Comparing the average luminosities shows that HBLs and FSRQs are different at $3 \sigma$, while HBLs and LBLs have similar $\left\langle L_{1 \mathrm{keV}}\right\rangle$.

Using published radio fluxes at $5 \mathrm{GHz}$, we derived the radio-to-X-ray indices $\alpha_{\mathrm{rx}}$. In Fig. 3 we plot the X-ray photon index from Table 4 versus $\alpha_{\mathrm{rx}}$. The blazars separate in two groups: HBLs have $\alpha_{\mathrm{rx}}<0.75$ and $\Gamma_{x}>2$, while LBLs and FSRQs have $\alpha_{\mathrm{rx}}>0.75$ and $\Gamma_{x}<2$. One LBL (BL Lac) and 4 FSRQs (WGA J0546.6-6415, RGB J1629+4008, RGB J1722+2436, and S5 2116+81) fill the gap between the two groups. As discussed in Padovani et al. 2002, these sources exhibit spectral energy distributions more similar to HBLs and were dubbed "High-energy peaked 


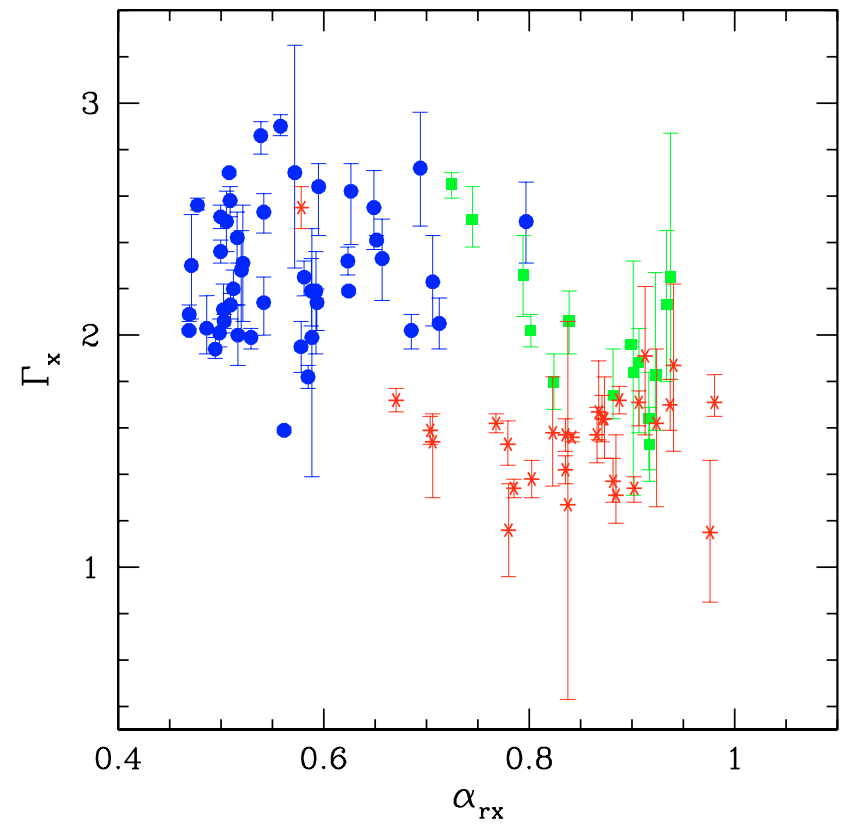

Fig. 3. X-ray photon indices vs. broad band radio to X-ray indexes. Circle: HBLs, square: LBLs, star: FSRQs.

FSRQs". It still remains to be demonstrated whether the steep soft X-ray spectrum of these sources is due to synchrotron from the jet or is thermal emission perhaps related to the high-energy tail of the disk Blue Bump (as, e.g., in 3C 273).

Figure 4 shows the plot of the radio luminosity versus the X-ray luminosity for HBLs, LBLs, and FSRQs. There is a clear correlation between the luminosities at the two wavelengths. However, different trends are observed between HBLs and LBLs-FSRQs: for a given X-ray luminosity, LBLs and FSRQs appear more luminous at radio.

To quantify the degree of linear correlation, we calculated the linear correlation coefficient $r$ and computed the chance probability $P_{\mathrm{r}}(N)$ that a random sample of $N$ uncorrelated pairs of measurements would yield a linear correlation coefficient equal or larger than $|r|$; if the chance probability is small, the two quantities are likely to be correlated. For HBLs, the linear correlation coefficient is $r=0.72$ and the chance probability $P_{\mathrm{r}}=2 \times 10^{-7}$. For LBLs-FSRQs, $r=0.90$ and $P=2.8 \times 10^{-15}$. From a linear fit to the data in Fig. 4 (i.e., considering the logarithmic values for the luminosities), we derive the following expressions: $\log L_{\mathrm{X}}=12.5+0.64 \times \log L_{\mathrm{R}}$ for HBLs, and $\log L_{X}=-3.9+1.06 \times \log L_{R}$ for LBLs-FSRQs. These results should be taken with caution, since our sample is very likely biased toward brighter X-ray sources.

In closing, we comment on flux and spectral variability. More details can be found in the publications for individual sources from the original PIs of the observations. Several sources in our sample were observed repeatedly with BeppoSAX. Inspection of the plots of flux versus spectral index shows that significant flux and spectral variability is present only for the HBL class, with the familiar trend of flatter slope with increasing flux. This confirms previous results based on various X-ray satellites (e.g., Mkn 421 with ASCA, Takahashi et al. 1999).

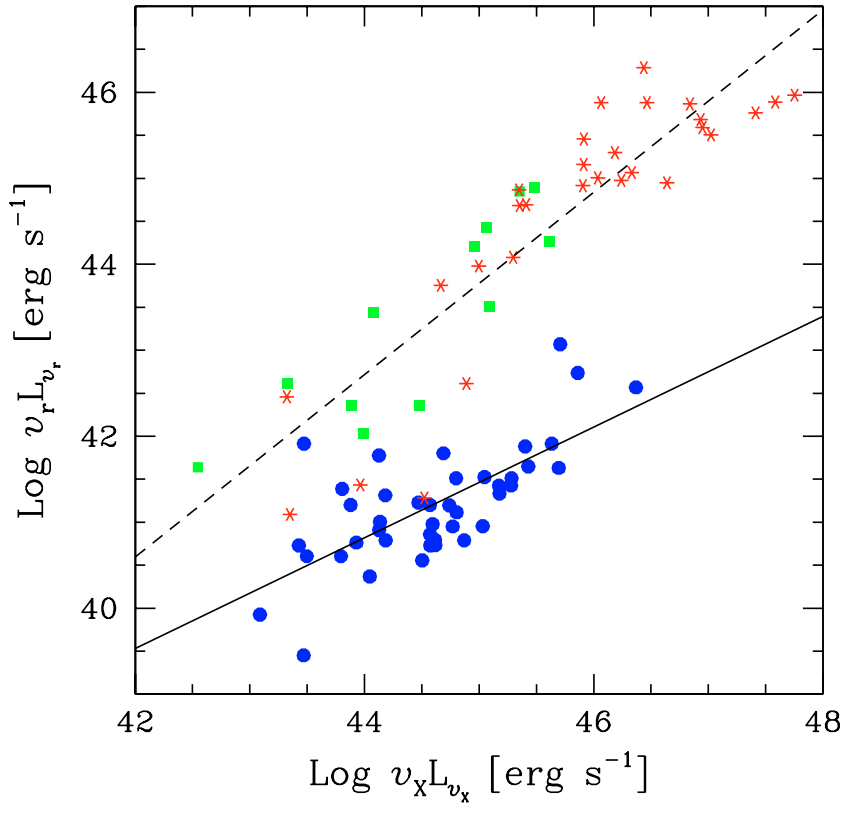

Fig. 4. Monochromatic X-ray (1 keV) luminosities vs. monochromatic radio $(5 \mathrm{GHz})$ luminosities. Circle: HBLs, square: LBLs, star: FSRQs. Linear correlations are plotted for the HBL class (continuous line) and for the LBL-FSRQ classes (dashed line).

In summary, we presented an analysis of the BeppoSAX database for blazars. Most HBLs have downward-curved continua, in agreement with the synchrotron interpretation, while the brightest FSRQs and LBLs show upward curvatures. While there is a net separation between HBLs on one side, and LBLs and FSRQs on the other, the distinction between LBLs and FSRQs is more blurry. Conceivably, this is due to optical identification ambiguities, as the classification of a higher-luminosity blazar as an LBL or FSRQ depends on the Equivalent Width of the optical emission lines; thus, variability of the optical continuum makes this classification highly dependent on the observation epoch.

Acknowledgements. We gratefully acknowledge financial support from NASA grants NAG5-10073 (D.D., R.M.S.) and LTSA grant NAG5-10708 (R.M.S., M.G.). R.M.S. was also supported by an NSF CAREER award and the Clare Boothe Luce Program of the Henry Luce Foundation. This research made use of data retrieved from the ASI/ASDC-BeppoSAXpublic archive and the NASA/IPAC Extragalactic Database (NED) which is operated by the Jet Propulsion Laboratory, Caltech, under contract with the National Aeronautics and Space Administration.

\section{References}

Boella, G., Butler, R. C., Perola, G. C., et al. 1997, A\&AS, 122, 299

Dickey, J. M., \& Lockman, F. J. 1990, ARA\&A, 28, 215

Donato, D., Ghisellini, G., Tagliaferri, G., \& Fossati, G. 2001, A\&A, 375,739

Fiore, F., Guainazzi, M., \& Grandi, P. 1999, Cookbook for NFI BeppoSAX Spectral Ananlysis v. 1.2 
Fossati, G., Maraschi, L., Celotti, A., Comastri, A., \& Ghisellini, G. 1997, MNRAS, 289, 136

Fossati, G., Celotti, A., Chiaberge, M., et al. 2000, ApJ, 541, 166

Giommi, P., \& Padovani, P. 1994, MNRAS, 268, 51

Giommi, P., Ansari, S. G., \& Micol, A. 1995, A\&AS, 109, 267

Giommi, P., Capalbi, M., Fiocchi, M., et al. 2002, Babs. Conf., 63

Mannheim, K. 1993, A\&A, 269, 67

Maraschi, L., Ghisellini, G., \& Celotti, A. 1992, ApJ, 397, L5

Padovani, P., \& Giommi, P. 1995, ApJ, 444, 567

Padovani, P., Costamante, L., Ghisellini, G., Giommi, P., \& Perlman, E. 2002, ApJ, 581, 895

Perlman, E. S., Stocke, J. T., Wang, Q. D., \& Morris, S. L. 1996, ApJ, 456,451
Sambruna, R. M., Barr, P., Giommi, P., et al. 1994a, ApJS, 95, 371 Sambruna, R. M., Barr, P., Giommi, P., et al. 1994b, ApJ, 434, 468 Sambruna, R. M., Maraschi, L., \& Urry, C. M. 1996, ApJ, 463, 444 Sambruna, R. M., Eracleous, M., \& Mushotzky, R. F. 1999, ApJ, 526, 60

Sikora, M., Begelman, M. C., \& Rees, M. J. 1994, ApJ, 421, 153

Takahashi, T., Tashiro, M., Madejski, G., et al. 1996, ApJ, 470, 89

Takahashi, T., Madejski, G., \& Kubo, H. 1999, APh, 11, 177

Tashiro, M., Makishima, K., Ohashi, T., et al. 1995, PASJ, 47, 131

Tavecchio, F., Maraschi, L., Pian, E., et al. 2001, ApJ, 554, 725

Urry, C. M., Sambruna, R. M., Worrall, D. M., et al. 1996, ApJ, 463, 424 
D. Donato et al.: A BeppoSAX spectral catalog of blazars, Online Material p 1

\section{Online Material}


Table 2. Observation Log.

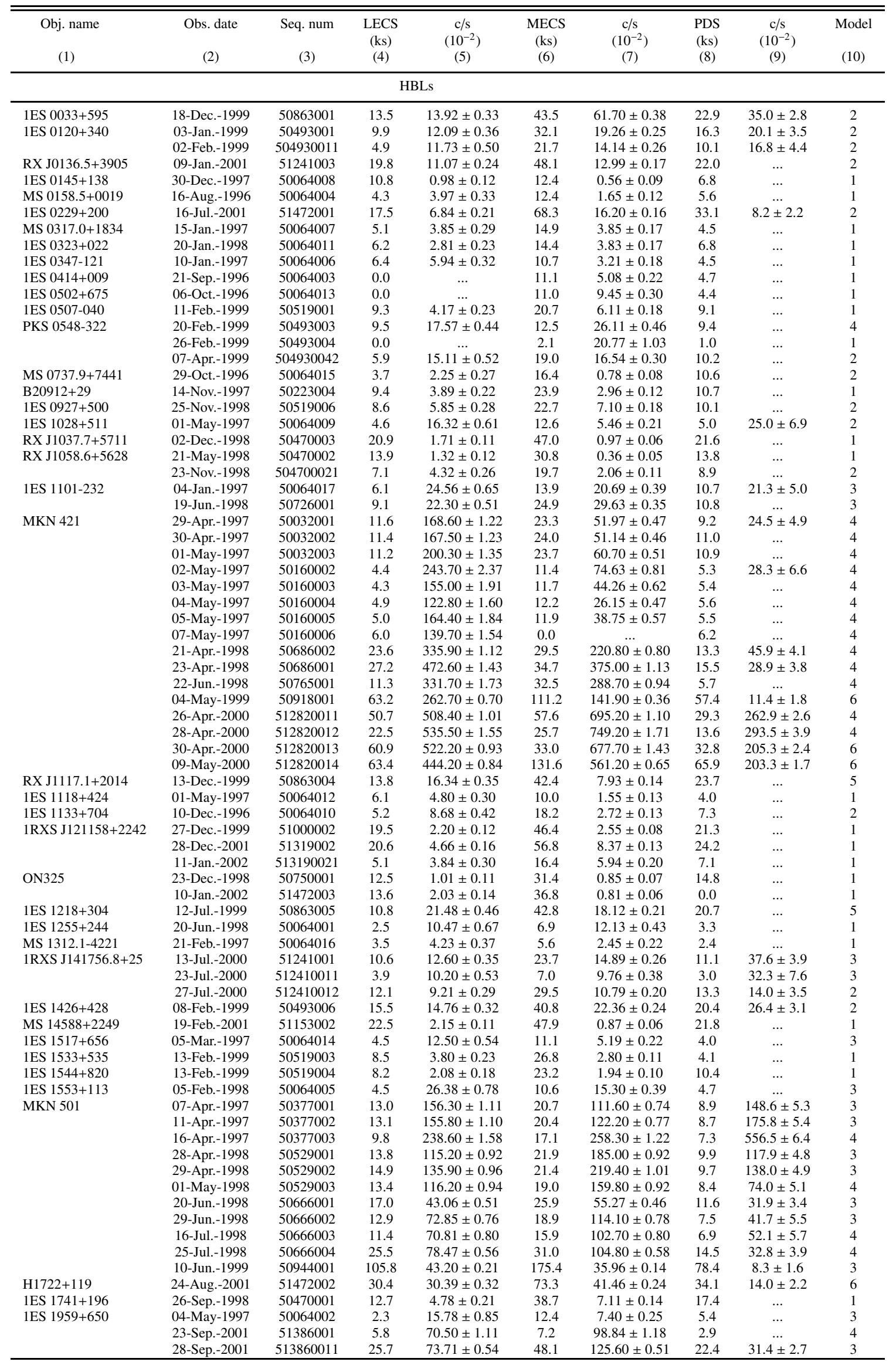


Table 2. continued.

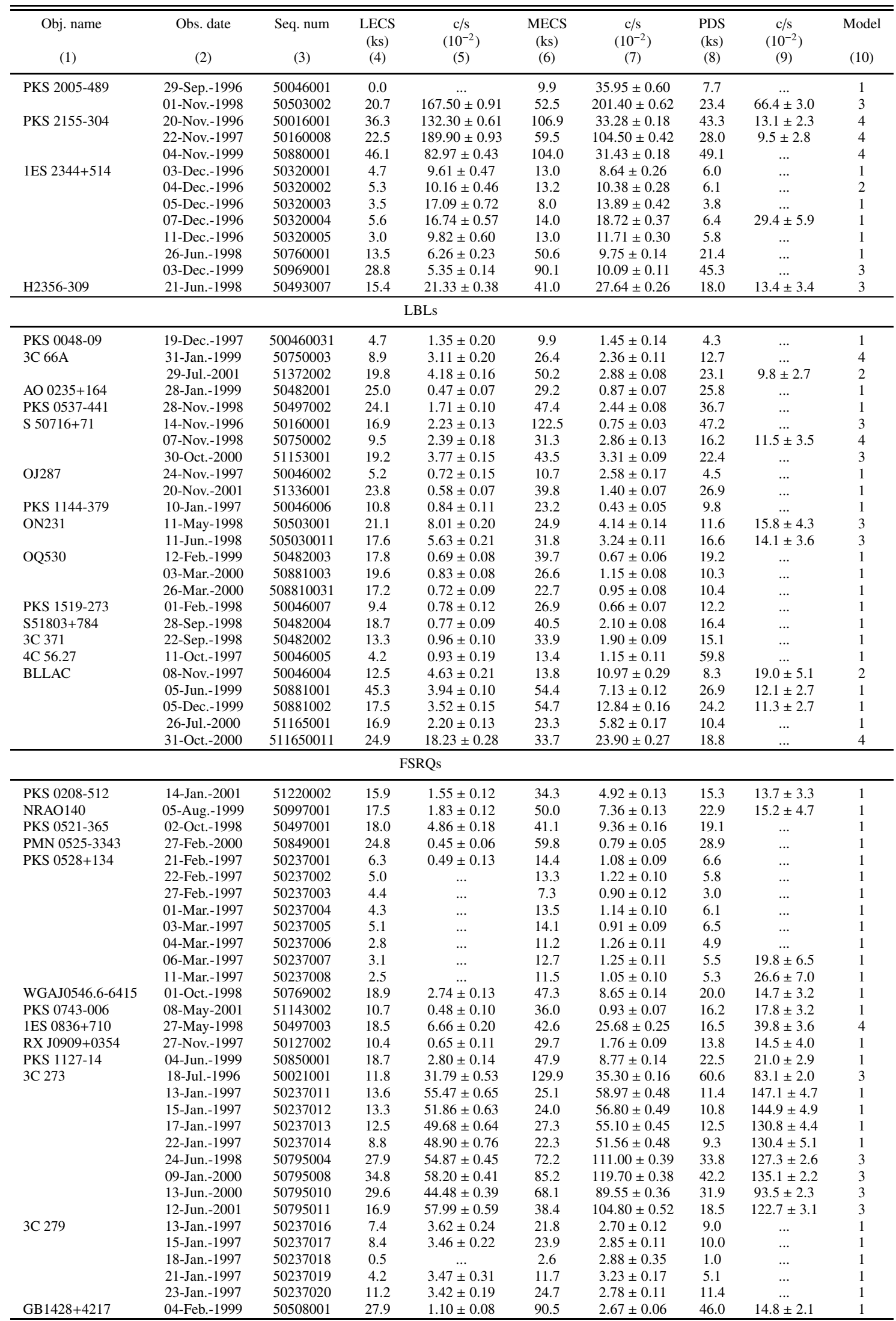


Table 2. continued.

\begin{tabular}{|c|c|c|c|c|c|c|c|c|c|}
\hline $\begin{array}{l}\text { Obj. name } \\
\text { (1) }\end{array}$ & $\begin{array}{l}\text { Obs. date } \\
\text { (2) }\end{array}$ & $\begin{array}{l}\text { Seq. num } \\
\text { (3) }\end{array}$ & $\begin{array}{c}\text { LECS } \\
(\mathrm{ks}) \\
(4)\end{array}$ & $\begin{array}{c}\mathrm{c} / \mathrm{s} \\
\left(10^{-2}\right) \\
(5)\end{array}$ & $\begin{array}{c}\text { MECS } \\
(\mathrm{ks}) \\
(6)\end{array}$ & $\begin{array}{c}\mathrm{c} / \mathrm{s} \\
\left(10^{-2}\right) \\
(7)\end{array}$ & $\begin{array}{c}\text { PDS } \\
(\mathrm{ks}) \\
(8)\end{array}$ & $\begin{array}{c}\mathrm{c} / \mathrm{s} \\
\left(10^{-2}\right) \\
(9)\end{array}$ & Model \\
\hline \multirow[t]{2}{*}{ GB1508+5714 } & 29-Mar.-1997 & 50127001 & 4.3 & $\ldots$ & 8.8 & $0.75 \pm 0.11$ & 3.9 & $\ldots$ & 1 \\
\hline & 01-Feb.-1998 & 501270011 & 7.0 & $\ldots$ & 17.5 & $0.51 \pm 0.08$ & 7.8 & & 1 \\
\hline PKS 1510-089 & 03-Aug.-1998 & 50497004 & 16.2 & $1.79 \pm 0.12$ & 43.9 & $4.99 \pm 0.11$ & 19.4 & $17.3 \pm 3.3$ & 3 \\
\hline RGB J1629+4008 & 11-Aug.-1999 & 50869001 & 21.2 & $3.28 \pm 0.14$ & 44.9 & $1.40 \pm 0.07$ & 22.8 & $\ldots$ & 1 \\
\hline 1ES $1641+399$ & 19-Feb.-1999 & 50727002 & 11.1 & $3.30 \pm 0.19$ & 25.9 & $5.08 \pm 0.15$ & 13.1 & $13.0 \pm 3.8$ & 1 \\
\hline RGB J1722+2436 & 13-Feb.-2000 & 50869002 & 12.4 & $0.71 \pm 0.10$ & 44.1 & $1.08 \pm 0.06$ & 19.7 & $\ldots$ & 1 \\
\hline $\mathrm{S} 41745+62$ & 29-Mar.-1997 & 50127006 & 58.4 & $0.41 \pm 0.04$ & 143.0 & $0.71 \pm 0.03$ & 59.8 & $\ldots$ & 1 \\
\hline \multirow[t]{2}{*}{ S52116+81 } & 29-Apr.-1998 & 50769001 & 13.4 & $7.54 \pm 0.25$ & 28.9 & $16.71 \pm 0.24$ & 13.3 & $15.9 \pm 4.0$ & 1 \\
\hline & 12-Oct.-1998 & 507690011 & 5.7 & $5.68 \pm 0.33$ & 19.7 & $11.88 \pm 0.25$ & 10.5 & $13.7 \pm 4.5$ & 1 \\
\hline PKS 2126-158 & 24-Мay-1999 & 51003001 & 79.9 & $3.62 \pm 0.07$ & 108.0 & $11.07 \pm 0.10$ & 51.6 & $10.5 \pm 2.0$ & 4 \\
\hline PKS 2134+004 & $25-N o v .-2000$ & 51143001 & 25.9 & $1.52 \pm 0.09$ & 75.1 & $2.14 \pm 0.06$ & 33.1 & $\ldots$ & 1 \\
\hline PKS 2149-306 & 31-Oct.-1997 & 50264001 & 17.9 & $2.74 \pm 0.14$ & 39.4 & $7.46 \pm 0.15$ & 16.7 & $13.0 \pm 3.7$ & 1 \\
\hline PKS $2223+210$ & 12-Nov.-1997 & 50127003 & 14.2 & $0.81 \pm 0.10$ & 18.4 & $2.09 \pm 0.12$ & 10.8 & $\ldots$ & 1 \\
\hline PKS 2223-05 & 10-Nov.-1997 & 50181006 & 9.4 & $0.79 \pm 0.12$ & 16.2 & $1.32 \pm 0.11$ & 6.9 & $\cdots$ & 1 \\
\hline \multirow[t]{5}{*}{$\mathrm{H} 2230+114$} & 11-Nov.-1997 & 50237021 & 11.0 & $2.03 \pm 0.15$ & 23.8 & $5.45 \pm 0.16$ & 11.6 & $\ldots$ & 1 \\
\hline & 13-Nov.-1997 & 50237022 & 10.3 & $2.91 \pm 0.19$ & 22.3 & $5.92 \pm 0.17$ & 10.2 & $\ldots$ & 1 \\
\hline & 16-Nov.-1997 & 50237023 & 13.2 & $1.91 \pm 0.14$ & 25.7 & $5.84 \pm 0.16$ & 12.1 & $13.7 \pm 4.3$ & 1 \\
\hline & 18-Nov.-1997 & 50237024 & 7.0 & $2.12 \pm 0.20$ & 12.2 & $5.63 \pm 0.22$ & 5.4 & $\ldots$ & 1 \\
\hline & 21-Nov.-1997 & 50237025 & 9.5 & $2.49 \pm 0.18$ & 19.4 & $5.98 \pm 0.18$ & 8.6 & $\begin{array}{l}\cdots \\
\ldots\end{array}$ & 1 \\
\hline PKS 2243-123 & 18-Nov.-1998 & 50727005 & 10.2 & $1.32 \pm 0.14$ & 27.5 & $1.89 \pm 0.09$ & 13.2 & $\ldots$ & 1 \\
\hline $\mathrm{H} 2251+158$ & 05-Jun.-2000 & 51220001 & 17.7 & $2.94 \pm 0.14$ & 48.5 & $10.92 \pm 0.15$ & 22.4 & $20.3 \pm 2.8$ & 1 \\
\hline
\end{tabular}

Columns: 1 = object name; 2 observation date; 3 = sequence number of the observation; 4 = exposure time for LECS; $5=$ counts rate for LECS in the energy range $0.1-2 \mathrm{keV} ; 6=$ exposure time for MECS (for the observations made before May 1997, MECS2 count-rates are considered); 7 = counts rate for MECS in the energy range $2-10 \mathrm{keV} ; 8=$ exposure time for PDS; $9=$ counts rate for PDS in the energy range 13-50 keV. Less than $3 \sigma$ detections are omitted; $10=$ model used for the best fit: $1=$ power law with absorption fixed at Galactic value; 2 = power law with free absorption; 3 = broken power law with absorption fixed at Galactic value; 4 = continuously curved parabola with absorption fixed at Galactic value; 5 = broken power law with free absorption; 6 = continuously curved parabola with free absorption. 
D. Donato et al.: A BeppoSAX spectral catalog of blazars, Online Material p 5

Table 3. Sources with power law as best-fit model.

\begin{tabular}{|c|c|c|c|c|c|c|c|}
\hline \multirow[b]{2}{*}{$\begin{array}{l}\text { Obj. name } \\
\text { (1) }\end{array}$} & \multirow[b]{2}{*}{$\begin{array}{l}\text { Obs. date } \\
\text { (2) }\end{array}$} & \multicolumn{2}{|c|}{$N_{\mathrm{H}}$ FIXED } & \multicolumn{3}{|c|}{$N_{\mathrm{H}}$ FREE } & \multirow[b]{2}{*}{$\begin{array}{c}\text { Flux } \\
(8)\end{array}$} \\
\hline & & $\begin{array}{l}\Gamma \\
(3)\end{array}$ & $\begin{array}{c}\chi_{r}^{2} / \text { d.o.f. } \\
\text { (4) }\end{array}$ & $\begin{array}{l}N_{\mathrm{H}} \\
(5)\end{array}$ & $\begin{array}{c}\Gamma \\
(6)\end{array}$ & $\begin{array}{c}\chi_{r}^{2} / \text { d.o.f. } \\
\text { (7) }\end{array}$ & \\
\hline \multicolumn{8}{|c|}{ HBLs } \\
\hline 1ES $0033+595$ & 18-Dec.-1999 & & & $35.5_{-7.6}^{+8.3}$ & $2.06_{-0.03}^{+0.03}$ & $1.22 / 155$ & 58.9 \\
\hline \multirow[t]{2}{*}{1 ES $0120+340$} & 03-Jan.-1999 & & & $9.3_{-2.4}^{-3.0 .3}$ & $2.13_{-0.05}^{+0.06}$ & $1.07 / 99$ & 17.1 \\
\hline & 02-Feb.-1999 & & & $5.6_{-25}^{+6.4}$ & $2.33_{-0.08}^{+0.09}$ & $0.84 / 39$ & 13.3 \\
\hline RX J0136.5+3905 & 09-Jan.-2001 & & & $7.9_{-2.5}^{+3.3}$ & $2.58_{-0.06}^{+0.07}$ & $1.27 / 99$ & 10.8 \\
\hline 1ES $0145+138$ & 30-Dec.-1997 & $1.99_{-0.47}^{+0.60}$ & $0.91 / 24$ & & & & 0.6 \\
\hline MS 0158.5+0019 & 16-Aug.-1996 & $2.28_{-0.17}^{+0.27}$ & $0.86 / 25$ & & & & 2.9 \\
\hline $1 \mathrm{ES} 0229+200$ & 16-Jul.-2001 & & & $10.0_{-41}^{+5.1}$ & $1.99_{-0.04}^{+0.05}$ & $1.03 / 99$ & 14.9 \\
\hline MS $0317.0+1834$ & 15-Jan.-1997 & $2.00_{-0.13}^{+0.13}$ & $0.98 / 59$ & & & & 7.1 \\
\hline $1 \mathrm{ES} 0323+022$ & 20-Jan.-1998 & $2.19_{-0.17}^{-0.13}$ & $0.60 / 32$ & & & & 3.5 \\
\hline 1ES 0347-121 & 10-Jan.-1997 & $2.03_{-0.14}^{+0.11}$ & $1.04 / 54$ & & & & 6.2 \\
\hline 1ES 0414+009 & 21-Sep.-1996 & $2.53_{-0.08}^{+0.09}$ & $0.94 / 54$ & & & & 8.7 \\
\hline 1ES $0502+675$ & 06-Oct.-1996 & $2.30_{-0.22}^{+0.20}$ & $1.13 / 97$ & & & & 19.2 \\
\hline 1ES 0507-040 & 11-Feb.-1999 & $2.14_{-0.11}^{+0.14}$ & $0.78 / 36$ & & & & 5.9 \\
\hline \multirow[t]{2}{*}{ PKS 0548-322 } & 26-Feb.-1999 & $2.28_{-0.22}^{+0.11}$ & $0.61 / 16$ & & & & 17.8 \\
\hline & 07-Apr.-1999 & & & $3.1_{-0.8}^{+0.8}$ & $2.25_{-0.07}^{+0.08}$ & $0.92 / 35$ & 15.7 \\
\hline MS0737.9+7441 & 29-Oct.-1996 & & & $27.3_{-18.0}^{+5.5 .5}$ & $2.70_{-0.55}^{+0.01}$ & $0.95 / 16$ & 1.4 \\
\hline B20912+29 & 14-Nov.-1997 & $2.05_{-0.11}^{+0.11}$ & $1.01 / 23$ & & & & 2.8 \\
\hline 1ES $0927+500$ & 25-Nov.-1998 & & & $3.3_{-1.1}^{+1.5}$ & $2.11_{-0.11}^{+0.10}$ & $0.96 / 33$ & 6.8 \\
\hline 1ES $1028+511$ & 01-May-1997 & & & $4.7_{-1.3}^{-1.1 .5}$ & $2.42_{-0.11}^{+0.11}$ & $0.91 / 91$ & 10.1 \\
\hline RX J1037.7+5711 & 02-Dec.-1998 & $2.23_{-0.19}^{+0.19}$ & $0.94 / 24$ & & & & 0.9 \\
\hline \multirow{2}{*}{ RX J1058.6+5628 } & 21-May-1998 & $2.26_{-0.54}^{+0.60}$ & $1.08 / 21$ & & & & 0.3 \\
\hline & 23-Nov.-1998 & & & $2.3_{-0.8}^{+1.3}$ & $2.72_{-0.24}^{+0.25}$ & $0.79 / 33$ & 1.7 \\
\hline 1ES $1118+424$ & 01-May-1997 & $2.19_{-0.14}^{+0.15}$ & $0.90 / 38$ & & & & 3.1 \\
\hline 1ES $1133+704$ & 10-Dec.-1996 & & & $2.0_{-0.8}^{+0.9}$ & $2.55_{-0.16}^{+0.18}$ & $0.85 / 39$ & 5.0 \\
\hline \multirow[t]{3}{*}{$\mathrm{RX} \mathrm{J1211.9+2242}$} & 27-Dec.-1999 & $1.93_{-0.10}^{+0.10}$ & $1.35 / 24$ & & & & 2.5 \\
\hline & 28-Dec.-2001 & $1.93_{-0.06}^{+0.06}$ & $1.04 / 90$ & & & & 7.6 \\
\hline & 11-Jan.-2002 & $2.05_{-0.12}^{+0.06}$ & $1.00 / 40$ & & & & 5.6 \\
\hline \multirow[t]{2}{*}{ ON325 } & 23-Dec.-1998 & $2.55_{-0.27}^{+0.31}$ & $1.32 / 25$ & & & & 0.6 \\
\hline & 10-Jan.-2002 & $2.49_{-0.17}^{+0.18}$ & $0.90 / 33$ & & & & 0.6 \\
\hline 1ES 1255+244 & 20-Jun.-1998 & $1.95_{-0.11}^{+0.11}$ & $0.79 / 24$ & & & & 11.7 \\
\hline MS 1312.1-4221 & 21-Feb.-1997 & $2.31_{-0.25}^{+0.11}$ & $0.80 / 16$ & & & & 4.6 \\
\hline 1RXS J141756.8+25 & 27-Jul.-2000 & & & $2.3_{-07}^{+0.8}$ & $2.20_{-0.08}^{+0.07}$ & $1.27 / 96$ & 9.3 \\
\hline 1ES $1426+428$ & 08-Feb.-1999 & & & $0.8_{-0.4}^{+0.4}$ & $1.94_{-0.05}^{+0.04}$ & $1.12 / 142$ & 20.3 \\
\hline MS $14588+2249$ & 19-Feb.-2001 & $2.62_{-012}^{+0.23}$ & $1.24 / 24$ & & & & 0.7 \\
\hline 1ES $1533+535$ & 13-Feb.-1999 & $2.14_{-0.06}^{+0.12}$ & $1.22 / 69$ & & & & 2.6 \\
\hline 1ES $1544+820$ & 13-Feb.-1999 & $2.64_{-0.10}^{+0.01}$ & $0.93 / 39$ & & & & 1.5 \\
\hline 1ES $1741+196$ & 26-Sep.-1998 & $2.02_{-0.07}^{+0.08}$ & $1.14 / 35$ & & & & 6.9 \\
\hline PKS 2005-489 & 29-Sep.-1996 & $2.36_{-0.05}^{+0.07}$ & $1.06 / 118$ & & & & 58.7 \\
\hline \multirow[t]{6}{*}{ 1ES $2344+514$} & 03-Dec.-1996 & $2.05_{-0.08}^{+0.09}$ & $0.93 / 39$ & & & & 17.2 \\
\hline & 04-Dec.-1996 & & & $13.3_{-60}^{+6.8}$ & $2.05_{-0.09}^{+0.08}$ & $1.10 / 134$ & 18.8 \\
\hline & 05-Dec.-1996 & $2.12_{-0.08}^{+0.09}$ & $1.59 / 51$ & & & & 27.1 \\
\hline & 07-Dec.-1996 & $1.82_{-0.05}^{+0.08}$ & $0.96 / 230$ & & & & 35.3 \\
\hline & 11-Dec.-1996 & $2.17_{-0.03}^{+0.03}$ & $1.15 / 144$ & & & & 20.4 \\
\hline & 26-Jun.-1998 & $2.32_{-0.06}^{+0.06}$ & $0.98 / 93$ & & & & 8.5 \\
\hline \multicolumn{8}{|c|}{ LBLs } \\
\hline PKS 0048-09 & 19-Dec.-1997 & $1.96_{-0.01}^{+0.01}$ & $1.11 / 13$ & & & & 1.4 \\
\hline $3 \mathrm{C} 66 \mathrm{~A}$ & 29-Jul.-2001 & & & $14.4_{-7.4}^{+7.6}$ & $2.40_{-0.13}^{+0.15}$ & $1.49 / 34$ & 2.7 \\
\hline AO $0235+164$ & 28-Jan.-1999 & $2.13_{-0.32}^{+0.33}$ & $1.15 / 26$ & & & & 0.7 \\
\hline PKS 0537-441 & 28-Nov.-1998 & $1.80_{-0.12}^{+0.12}$ & $0.66 / 24$ & & & & 2.4 \\
\hline \multirow{2}{*}{ OJ 287} & 24-Nov.-1997 & $1.95_{-0.29}^{+0.12}$ & $0.88 / 24$ & & & & 2.4 \\
\hline & 20-Nov.-2001 & $1.64_{-0.20}^{+0.29}$ & $0.93 / 51$ & & & & 1.3 \\
\hline PKS 1144-379 & 10-Jan.-1997 & $1.83_{-0.44}^{+0.24}$ & $0.88 / 26$ & & & & 1.1 \\
\hline OQ530 & 12-Feb.-1999 & $1.92_{-0.29}^{+0.29}$ & $0.86 / 26$ & & & & 0.6 \\
\hline & 03-Mar.-2000 & $1.53_{-0.32}^{+0.29}$ & $1.06 / 39$ & & & & 1.1 \\
\hline & 26-Mar.-2000 & $1.88_{-0.15}^{+0.30}$ & $0.83 / 32$ & & & & 0.8 \\
\hline PKS 1519-273 & 01-Feb.-1998 & $2.25_{-0.62}^{+0.43}$ & $1.46 / 16$ & & & & 0.5 \\
\hline S51803+784 & 28-Sep.-1998 & $1.53_{-0.16}^{-0.02}$ & $1.04 / 22$ & & & & 2.2 \\
\hline 3C 371 & 22-Sep.-1998 & $1.74_{-0.20}^{+0.10}$ & $1.14 / 51$ & & & & 1.7 \\
\hline 4C 56.27 & 11-Oct.-1997 & $1.84_{-0.48}^{+0.20}$ & $0.97 / 20$ & & & & 1.1 \\
\hline
\end{tabular}


D. Donato et al.: A BeppoSAX spectral catalog of blazars, Online Material p 6

Table 3. continued.

\begin{tabular}{|c|c|c|c|c|c|c|c|}
\hline \multirow[b]{2}{*}{$\begin{array}{l}\text { Obj. name } \\
\text { (1) }\end{array}$} & \multirow[b]{2}{*}{$\begin{array}{l}\text { Obs. date } \\
\text { (2) }\end{array}$} & \multicolumn{2}{|c|}{$N_{\mathrm{H}}$ FIXED } & \multicolumn{3}{|c|}{$N_{\mathrm{H}}$ FREE } & \multirow[b]{2}{*}{$\begin{array}{c}\text { Flux } \\
(8)\end{array}$} \\
\hline & & $\begin{array}{c}\Gamma \\
(3)\end{array}$ & $\begin{array}{l}\chi_{r}^{2} / \text { d.o.f. } \\
\text { (4) }\end{array}$ & $\begin{array}{l}N_{\mathrm{H}} \\
(5)\end{array}$ & $\begin{array}{l}\Gamma \\
(6)\end{array}$ & $\begin{array}{c}\chi_{r}^{2} / \text { d.o.f. } \\
\text { (7) }\end{array}$ & \\
\hline \multirow[t]{4}{*}{ BLLAC } & 08-Nov.-1997 & & & $11.0_{-6.9}^{+8.4}$ & $1.88_{-0.12}^{+0.06}$ & $1.11 / 33$ & 11.1 \\
\hline & 05-Jun.-1999 & $2.02_{-0.07}^{+0.07}$ & $1.12 / 93$ & & & & 6.5 \\
\hline & 05-Dec.-1999 & $1.59_{-0.05}^{+0.05}$ & $0.99 / 93$ & & & & 12.7 \\
\hline & 26-Jul.-2000 & $1.88_{-0.12}^{+0.12}$ & $1.21 / 24$ & & & & 5.8 \\
\hline \multicolumn{8}{|c|}{ FSRQs } \\
\hline PKS 0208-512 & 14-Jan.-2001 & $1.64_{-0.10}^{+0.10}$ & $1.33 / 93$ & & & & 4.5 \\
\hline NRAO140 & 05-Aug.-1999 & $1.57_{-0.07}^{+0.07}$ & $1.26 / 93$ & & & & 7.2 \\
\hline PKS 0521-365 & 02-Oct.-1998 & $1.72^{+0.006}$ & $1.00 / 147$ & & & & 8.7 \\
\hline PMN 0525-3343 & 27-Feb.-2000 & $1.58_{-0.24}^{+0.023}$ & $0.99 / 52$ & & & & 0.7 \\
\hline \multirow[t]{8}{*}{ PKS 0528+134 } & 21-Feb.-1997 & $1.28_{-0.26}^{+0.24}$ & $0.94 / 27$ & & & & 2.7 \\
\hline & 22-Feb.-1997 & $1.26_{-030}^{+0.28}$ & $0.45 / 24$ & & & & 2.9 \\
\hline & 27-Feb.-1997 & $1.40_{-0.57}^{+0.50}$ & $0.94 / 14$ & & & & 2.1 \\
\hline & 01-Mar-1997 & $1.34_{-0.29}^{+0.029}$ & $1.14 / 25$ & & & & 3.3 \\
\hline & 03-Mar--1997 & $\begin{array}{r}-0.29 \\
1.15_{-0.31}^{+0.30}\end{array}$ & $1.25 / 24$ & & & & 1.9 \\
\hline & 04-Mar-1997 & $1.28_{-0.34}^{+0.33}$ & $0.76 / 20$ & & & & 2.6 \\
\hline & 06-Mar--1997 & $1.12_{-026}^{+0.26}$ & $1.09 / 23$ & & & & 2.9 \\
\hline & 11-Mar--1997 & $1.62_{-032}^{+0.36}$ & $1.48 / 21$ & & & & 2.2 \\
\hline WGAJ0546.6-6415 & 01-Oct.-1998 & $1.59_{-0.06}^{+0.06}$ & $1.87 / 93$ & & & & 8.2 \\
\hline PKS 0743-006 & 08-May-2001 & $1.91_{-0.30}^{-0.066}$ & $0.81 / 35$ & & & & 0.9 \\
\hline RGB J0909+039 & 27-Nov.-1997 & $\begin{array}{r}-0.00 \\
1.16_{-0.20}^{+0.20}\end{array}$ & $0.73 / 42$ & & & & 1.9 \\
\hline PKS 1127-14 & 04-Jun.-1999 & $1.42_{-0.06}^{+0.06}$ & $0.94 / 93$ & & & & 8.7 \\
\hline \multirow[t]{4}{*}{$3 \mathrm{C} 273$} & 13-Jan.-1997 & $1.56_{-0.02}^{+0.000}$ & $0.91 / 231$ & & & & 117.0 \\
\hline & 15-Jan.-1997 & $1.58_{-0.02}^{+0.02}$ & $1.19 / 231$ & & & & 112.0 \\
\hline & 17-Jan.-1997 & $1.62_{-0.02}^{+0.02}$ & $1.35 / 231$ & & & & 108.0 \\
\hline & 22-Jan.-1997 & $1.55_{-0.02}^{+0.022}$ & $0.97 / 231$ & & & & 103.0 \\
\hline \multirow[t]{5}{*}{$3 \mathrm{C} 279$} & 13-Jan.-1997 & $1.67_{-0.12}^{-0.022}$ & $0.98 / 39$ & & & & 5.6 \\
\hline & 15-Jan.-1997 & $1.70_{-0.11}^{-0.0 .12}$ & $0.75 / 50$ & & & & 5.8 \\
\hline & 18-Jan.-1997 & $1.43_{-0.47}^{+0.047}$ & $1.00 / 13$ & & & & 7.4 \\
\hline & 21-Jan.-1997 & $1.53_{-0.16}^{+0.16}$ & $0.79 / 48$ & & & & 6.4 \\
\hline & 23-Jan.-1997 & $1.59^{+0.10}$ & $1.18 / 50$ & & & & 5.7 \\
\hline GB1428+4217 & 04-Feb.-1999 & $1.53_{-010}^{+0.09}$ & $1.15 / 35$ & & & & 2.8 \\
\hline \multirow[t]{2}{*}{ GB1508+5714 } & 29-Mar-1997 & $\begin{array}{r}-.10 \\
0.65_{-0.85}^{+0.83}\end{array}$ & $0.84 / 14$ & & & & 1.3 \\
\hline & 01-Feb.-1998 & $1.27_{-0.79}^{+0.84}$ & $1.33 / 18$ & & & & 0.5 \\
\hline RGB J1629+4008 & 11-Aug.-1999 & $\begin{array}{r}-0.19 \\
2.55_{-0.09}^{+0.09}\end{array}$ & $1.19 / 35$ & & & & 1.3 \\
\hline 1ES $1641+399$ & 19-Feb.-1999 & $1.71_{-0.05}^{+0.099}$ & $1.92 / 35$ & & & & 5.1 \\
\hline RGB J1722+2436 & 13-Feb.-2000 & $1.54_{-0.12}^{+0.024}$ & $1.00 / 44$ & & & & 1.0 \\
\hline S41745+62 & 29-Mar-1997 & $1.64_{-0.17}^{+0.17}$ & $0.94 / 97$ & & & & 0.8 \\
\hline \multirow[t]{2}{*}{ S52116+81 } & 29-Apr--1998 & $1.72_{-0.05}^{-0.0 .18}$ & $1.23 / 93$ & & & & 15.8 \\
\hline & 12-Oct.-1998 & $1.83_{-0.09}^{+0.099}$ & $1.25 / 35$ & & & & 11.9 \\
\hline PKS 2134+004 & 25-Nov.-2000 & $1.71_{-0.12}^{-0.099}$ & $1.69 / 22$ & & & & 2.2 \\
\hline PKS 2149-306 & 31-Oct.-1997 & $1.38_{-0.08}^{-0.0 .12}$ & $1.13 / 32$ & & & & 8.1 \\
\hline PKS 2223+210 & 12-Nov.-1997 & $1.31_{-0.12}^{-0.012}$ & $0.87 / 37$ & & & & 2.2 \\
\hline PKS 2223-05 & 10-Nov.-1997 & $\begin{array}{r}-0.00 \\
1.87_{-0.35}^{+0.37}\end{array}$ & $1.17 / 15$ & & & & 1.3 \\
\hline \multirow[t]{5}{*}{$\mathrm{H} 2230+114$} & 11-Nov.-1997 & $1.57_{-012}^{+0.12}$ & $0.87 / 35$ & & & & 5.6 \\
\hline & 13-Nov.-1997 & $1.50_{-011}^{+0.11}$ & $0.95 / 35$ & & & & 6.2 \\
\hline & 16-Nov.-1997 & $1.47_{-010}^{+0.11}$ & $0.97 / 35$ & & & & 6.2 \\
\hline & 18-Nov.-1997 & $1.49_{-0.16}^{-0.110}$ & $1.27 / 41$ & & & & 5.5 \\
\hline & 21-Nov.-1997 & $1.50_{-0.13}^{-0.116}$ & $1.16 / 35$ & & & & 6.2 \\
\hline PKS 2243-123 & 18-Nov.-1998 & $\begin{array}{r}-.13 \\
1.67_{-0.22}^{+0.12}\end{array}$ & $0.97 / 44$ & & & & 1.8 \\
\hline $\mathrm{H} 2251+158$ & 05-Jun.-2000 & $\begin{array}{l}-0.022 \\
1.34_{-0.05}^{+0.06}\end{array}$ & $1.31 / 93$ & & & & 11.2 \\
\hline
\end{tabular}

Columns: 1 = object name; 2 = observation date; 3 = photon spectral index; 4 = reduced $\chi^{2}$ with degrees of freedom. Columns 3 and 4 refer to fits obtained with intrinsic absorption fixed to the Galactic value; $5=$ intrinsic absorption (in units of $10^{20} \mathrm{~cm}^{-2}$ ); $6=$ photon spectral index; $7=$ reduced $\chi^{2}$ with degrees of freedom. Columns 5 to 7 refer to fits obtained with intrinsic absorption free to vary; $8=\mathrm{X}$-ray unabsorbed flux in the energy range $2-10 \mathrm{keV}$ (in units of $10^{-12} \mathrm{erg} \mathrm{cm}^{-2} \mathrm{~s}^{-1}$ ). 
D. Donato et al.: A BeppoSAX spectral catalog of blazars, Online Material p 7

Table 4. Spectra best-fitted by a curved model.

\begin{tabular}{|c|c|c|c|c|c|c|c|c|c|c|c|}
\hline \multirow[b]{2}{*}{$\begin{array}{l}\text { Obj. name } \\
\text { (1) }\end{array}$} & \multirow[b]{2}{*}{$\begin{array}{l}\text { Obs. date } \\
\text { (2) }\end{array}$} & \multicolumn{4}{|c|}{$N_{\mathrm{H}}$ FIXED } & \multicolumn{5}{|c|}{$N_{\mathrm{H}}$ FREE } & \multirow[b]{2}{*}{$\begin{array}{l}\text { Flux } \\
(12)\end{array}$} \\
\hline & & $\begin{array}{l}\chi_{r}^{2} / \text { d.o.f. } \\
\text { (3) }\end{array}$ & $\begin{array}{l}\Gamma_{1} \\
\text { (4) }\end{array}$ & $\begin{array}{l}E_{\text {break }} \\
(5)\end{array}$ & $\begin{array}{l}\Gamma_{2} \\
(6)\end{array}$ & $\begin{array}{c}\chi_{r}^{2} / \text { d.o.f. } \\
\text { (7) }\end{array}$ & $\begin{array}{l}N_{\mathrm{H}} \\
(8)\end{array}$ & $\begin{array}{l}\Gamma_{1} \\
(9)\end{array}$ & $\begin{array}{c}E_{\text {break }} \\
(10)\end{array}$ & $\begin{array}{c}\Gamma_{2} \\
(11)\end{array}$ & \\
\hline \multicolumn{12}{|c|}{ HBLs } \\
\hline PKS 0548-322 & 20-Feb.-1999 & $1.21 / 91$ & $1.66_{-0.09}^{+0.13}$ & $0.58_{-0.58}^{+99.42}$ & $2.45_{-0.15}^{+0.40}$ & & & & & & 22.3 \\
\hline \multirow[t]{2}{*}{ 1ES 1101-232 } & 04-Jan.-1997 & $0.91 / 254$ & $1.73_{-0.09}^{+0.13}$ & $3.45_{-0.52}^{+0.06}$ & $2.19_{-0.11}^{+0.14}$ & & & & & & 36.8 \\
\hline & 19-Jun.-1998 & $1.02 / 88$ & $1.77_{-0.24}^{+0.16}$ & $1.25_{-0.25}^{+0.43}$ & $2.28_{-0.05}^{+0.05}$ & & & & & & 25.6 \\
\hline \multirow[t]{16}{*}{ Mkn 421} & 29-Apr.-1997 & $1.14 / 229$ & $2.26_{-0.04}^{+0.04}$ & $0.14_{-0.12}^{+0.59}$ & $3.05_{-0.07}^{+0.09}$ & & & & & & 84.5 \\
\hline & 30-Apr.-1997 & $0.79 / 229$ & $2.25_{-0.04}^{+0.04}$ & $0.25_{-0.22}^{+0.88}$ & $3.10_{-0.08}^{+0.09}$ & & & & & & 83.2 \\
\hline & 01-Мay-1997 & $1.10 / 229$ & $2.23_{-0.03}^{+0.04}$ & $0.16_{-0.15}^{+0.57}$ & $3.02_{-0.07}^{+0.08}$ & & & & & & 99.2 \\
\hline & 02-Мay-1997 & $1.09 / 229$ & $2.22_{-0.05}^{+0.05}$ & $0.22_{-0.21}^{+1.31}$ & $3.08_{-0.09}^{+0.11}$ & & & & & & 121.0 \\
\hline & 03-May-1997 & $1.07 / 229$ & $2.21_{-0.04}^{+0.06}$ & $3.29_{-1.85}^{+5.95}$ & $3.34_{-0.22}^{+0.32}$ & & & & & & 70.3 \\
\hline & 04-Мay-1997 & $1.07 / 229$ & $2.49_{-0.08}^{+0.06}$ & $0.16_{-0.15}^{+2.59}$ & $3.38_{-0.13}^{+0.20}$ & & & & & & 40.1 \\
\hline & 05-May-1997 & $1.27 / 216$ & $2.39_{-0.02}^{+0.02}$ & $0.18_{-0.09}^{+0.15}$ & $3.22_{-0.05}^{+0.05}$ & & & & & & 60.7 \\
\hline & 07-Мay-1997 & $0.85 / 42$ & $2.32_{-0.06}^{+0.07}$ & $2.02_{-1.33}^{+0.93}$ & $3.63_{-0.83}^{+0.37}$ & & & & & & 37.8 \\
\hline & 21-Apr--1998 & $0.95 / 146$ & $2.17_{-0.02}^{+0.02}$ & $1.14_{-0.63}^{+1.40}$ & $3.00_{-0.05}^{+0.05}$ & & & & & & 178.0 \\
\hline & 23-Apr.-1998 & $1.12 / 146$ & $2.02_{-0.02}^{+0.02}$ & $1.18_{-0.13}^{+0.15}$ & $2.64_{-0.02}^{+0.02}$ & & & & & & 312.0 \\
\hline & 22-Jun.-1998 & $1.45 / 133$ & $2.05_{-0.02}^{+0.02}$ & $0.43_{-0.32}^{+0.92}$ & $2.74_{-0.05}^{+0.05}$ & & & & & & 240.0 \\
\hline & 04-Мay-1999 & & & & & $1.89 / 145$ & $0.6_{-0.1}^{+0.1}$ & $2.36_{-0.02}^{+0.01}$ & $1.80_{-0.16}^{+0.20}$ & $3.02_{-0.02}^{+0.03}$ & 111.0 \\
\hline & 26-Apr--2000 & $2.58 / 146$ & $1.73_{-0.01}^{+0.01}$ & $1.39_{-0.30}^{+0.30}$ & $2.22_{-0.01}^{+0.01}$ & & & & & & 619.0 \\
\hline & 28-Apr.-2000 & $1.93 / 145$ & $1.73_{-0.01}^{+0.01}$ & $1.45_{-0.75}^{+1.38}$ & $2.23_{-0.02}^{+0.02}$ & & & & & & 669.0 \\
\hline & 30-Apr--2000 & & & & & $2.82 / 145$ & $0.3_{-0.3}^{+1.4}$ & $1.79_{-0.01}^{+0.01}$ & $4.57_{-0.77}^{+0.78}$ & $2.35_{-0.02}^{+0.02}$ & 598.0 \\
\hline & 09-Мay-2000 & & & & & $3.95 / 140$ & $0.3_{-0.3}^{+0.9}$ & $1.84_{-0.01}^{+0.01}$ & $2.12_{-0.14}^{+0.14}$ & $2.19_{-0.01}^{+0.01}$ & 497.0 \\
\hline RX J1117.1+2014 & 13-Dec.-1999 & & & & & $1.12 / 83$ & $1.5_{-0.8}^{+0.7}$ & $2.49_{-0.22}^{+0.16}$ & $1.52_{-0.39}^{+0.90}$ & $2.98_{-0.08}^{+0.09}$ & 6.1 \\
\hline 1ES 1218+304 & 12-Jul.-1999 & & & & & $0.77 / 93$ & $1.5_{-0.8}^{+0.7}$ & $2.21_{-0.13}^{+0.13}$ & $2.57_{-1.18}^{+1.59}$ & $2.57_{-0.06}^{+0.26}$ & 14.9 \\
\hline \multirow[t]{2}{*}{ 1RXS J141756.8+25 } & 13-Jul.-2000 & $0.95 / 96$ & $1.79_{-0.08}^{+0.07}$ & $3.00_{-0.68}^{+0.48}$ & $2.40_{-0.14}^{+0.14}$ & & & & & & 12.6 \\
\hline & 23-Jul.-2000 & $0.86 / 35$ & $1.74_{-0.18}^{+0.22}$ & $3.10_{-0.66}^{+0.54}$ & $2.60_{-0.27}^{+0.34}$ & & & & & & 8.7 \\
\hline 1ES 1517+656 & 05-Mar--1997 & $1.45 / 39$ & $1.40_{-0.24}^{+0.19}$ & $1.23_{-0.18}^{+0.19}$ & $2.60_{-0.07}^{+0.15}$ & & & & & & 9.5 \\
\hline 1ES $1553+113$ & 05-Feb.-1998 & $0.98 / 30$ & $1.96_{-0.21}^{+0.19}$ & $1.01_{-0.18}^{+0.46}$ & $2.79_{-0.11}^{+0.12}$ & & & & & & 13.7 \\
\hline \multirow[t]{11}{*}{ Mkn 501} & 07-Apr.-1997 & $0.97 / 234$ & $1.50_{-0.03}^{+0.04}$ & $1.08_{-0.08}^{+0.09}$ & $1.92_{-0.02}^{+0.02}$ & & & & & & 208.0 \\
\hline & 11-Apr.-1997 & $1.04 / 233$ & $1.48_{-0.02}^{+0.02}$ & $1.08_{-0.08}^{+0.09}$ & $1.92_{-0.02}^{+0.02}$ & & & & & & 232.0 \\
\hline & 16-Apr.-1997 & $1.00 / 229$ & $1.41_{-0.02}^{+0.02}$ & $2.88_{-1.02}^{+1.33}$ & $1.69_{-0.02}^{+0.02}$ & & & & & & 510.0 \\
\hline & 28-Apr.-1998 & $1.19 / 142$ & $1.54_{-0.04}^{+0.04}$ & $1.14_{-0.14}^{+0.13}$ & $1.85_{-0.02}^{+0.02}$ & & & & & & 172.0 \\
\hline & 29-Apr.-1998 & $1.02 / 140$ & $1.51_{-0.03}^{+0.03}$ & $1.26_{-0.11}^{+0.12}$ & $1.85_{-0.02}^{+0.02}$ & & & & & & 204.0 \\
\hline & 01-Мay-1998 & $1.09 / 143$ & $1.70_{-0.03}^{+0.04}$ & $2.65_{-1.62}^{+4.27}$ & $2.24_{-0.09}^{-0.12}$ & & & & & & 143.0 \\
\hline & 20-Jun.-1998 & $0.80 / 143$ & $1.72_{-0.05}^{+0.04}$ & $1.46_{-0.18}^{+0.38}$ & $2.08_{-0.03}^{+0.03}$ & & & & & & 49.2 \\
\hline & 29-Jun.-1998 & $1.00 / 145$ & $1.65_{-0.03}^{+0.03}$ & $2.71_{-0.30}^{+0.46}$ & $2.05_{-0.04}^{+0.07}$ & & & & & & 103.0 \\
\hline & 16-Jul.-1998 & $1.10 / 146$ & $1.69_{-0.05}^{+0.06}$ & $1.52_{-0.46}^{+0.73}$ & $2.23_{-0.06}^{+0.09}$ & & & & & & 90.5 \\
\hline & 25-Jul.-1998 & $1.02 / 146$ & $1.73_{-0.03}^{+0.03}$ & $1.59_{-0.29}^{+0.41}$ & $2.17_{-0.03}^{+0.04}$ & & & & & & 92.8 \\
\hline & 10-Jun.-1999 & $1.09 / 146$ & $2.00_{-0.02}^{+0.02}$ & $1.12_{-0.06}^{+0.06}$ & $2.45_{-0.02}^{+0.02}$ & & & & & & 30.2 \\
\hline H1722+119 & 24-Aug.-2001 & & & & & $1.19 / 145$ & $11.8_{-1.3}^{+1.3}$ & $2.52_{-0.08}^{+0.03}$ & $6.90_{-2.72}^{+0.74}$ & $3.21_{-0.65}^{+0.53}$ & 35.2 \\
\hline \multirow[t]{3}{*}{ 1ES 1959+650 } & 04-May-1997 & $1.02 / 45$ & $1.71_{-1.12}^{+0.42}$ & $1.23_{-0.37}^{+0.68}$ & $2.67_{-0.11}^{+0.11}$ & & & & & & 13.8 \\
\hline & 23-Sep.-2001 & $1.35 / 146$ & $1.94_{-0.13}^{+0.11}$ & $0.33_{-0.33}^{+0.31}$ & $2.50_{-0.05}^{+0.05}$ & & & & & & 83.4 \\
\hline & 28-Sep.-2001 & $1.44 / 143$ & $1.35_{-0.12}^{+0.10}$ & $1.11_{-0.05}^{+0.06}$ & $2.31_{-0.02}^{+0.02}$ & & & & & & 106.0 \\
\hline PKS 2005-489 & 01-Nov.-1998 & $1.74 / 146$ & $2.13_{-0.02}^{+0.02}$ & $2.59_{-0.88}^{+2.29}$ & $2.22_{-0.02}^{+0.02}$ & & & & & & 177.0 \\
\hline \multirow[t]{3}{*}{ PKS 2155-304 } & 20-Nov.-1996 & $0.95 / 228$ & $2.36_{-0.02}^{+0.02}$ & $1.55_{-0.20}^{+0.27}$ & $2.74_{-0.02}^{+0.03}$ & & & & & & 55.0 \\
\hline & 22-Nov.-1997 & $1.40 / 145$ & $2.28_{-0.02}^{+0.01}$ & $1.77_{-0.19}^{+0.22}$ & $2.91_{-0.03}^{+0.04}$ & & & & & & 83.3 \\
\hline & 04-Nov.-1999 & $1.07 / 145$ & $2.59_{-0.01}^{-0.02}$ & $2.22_{-0.35}^{+0.79}$ & $3.00_{-0.04}^{-0.09}$ & & & & & & 24.7 \\
\hline 1ES $2344+514$ & 03-Dec.-1999 & $1.48 / 91$ & $1.72_{-0.78}^{+0.19}$ & $1.58_{-0.60}^{+1.05}$ & $2.30_{-0.05}^{+0.05}$ & & & & & & 8.9 \\
\hline H2356-309 & 21-Jun.-1998 & $1.21 / 145$ & $1.56_{-0.07}^{+0.11}$ & $1.04_{-0.12}^{+0.30}$ & $2.11_{-0.04}^{+0.04}$ & & & & & & 24.3 \\
\hline
\end{tabular}


Table 4. continued.

\begin{tabular}{|c|c|c|c|c|c|c|c|c|c|c|c|}
\hline \multirow[b]{2}{*}{$\begin{array}{l}\text { Obj. name } \\
\text { (1) }\end{array}$} & \multirow[b]{2}{*}{$\begin{array}{l}\text { Obs. date } \\
\text { (2) }\end{array}$} & \multicolumn{4}{|c|}{$N_{\mathrm{H}}$ FIXED } & \multicolumn{5}{|c|}{$N_{\mathrm{H}}$ FREE } & \multirow[b]{2}{*}{$\begin{array}{l}\text { Flux } \\
\text { (12) }\end{array}$} \\
\hline & & $\begin{array}{l}\chi_{r}^{2} / \text { d.o.f. } \\
\text { (3) }\end{array}$ & $\begin{array}{l}\Gamma_{1} \\
(4)\end{array}$ & $\begin{array}{c}E_{\text {break }} \\
(5)\end{array}$ & $\begin{array}{l}\Gamma_{2} \\
(6)\end{array}$ & $\begin{array}{c}\chi_{r}^{2} / \text { d.o.f. } \\
\text { (7) }\end{array}$ & $\begin{array}{l}N_{\mathrm{H}} \\
(8)\end{array}$ & $\begin{array}{l}\Gamma_{1} \\
(9)\end{array}$ & $\begin{array}{c}E_{\text {break }} \\
(10)\end{array}$ & $\begin{array}{l}\Gamma_{2} \\
(11)\end{array}$ & \\
\hline \multicolumn{12}{|c|}{ LBLs } \\
\hline $3 \mathrm{C} 66 \mathrm{~A}$ & 31-Jan.-1999 & $1.31 / 33$ & $2.20_{-0.27}^{+0.12}$ & $0.26_{-1.07}^{+1.54}$ & $2.32_{-0.15}^{+0.15}$ & & & & & & 2.2 \\
\hline \multirow[t]{3}{*}{ S50716+71 } & 14-Nov.-1996 & $1.07 / 48$ & $2.70_{-0.14}^{-0.13}$ & $2.35_{-1.23}^{+0.80}$ & $1.96_{-0.22}^{+0.16}$ & & & & & & 1.5 \\
\hline & 07-Nov.-1998 & $1.92 / 33$ & $2.36_{-0.37}^{+0.22}$ & $1.75_{-1.75}^{+3.99}$ & $1.84_{-0.85}^{+0.26}$ & & & & & & 2.7 \\
\hline & 30-Oct.-2000 & $0.98 / 33$ & $2.82_{-0.12}^{+0.11}$ & $2.73_{-1.49}^{+0.42}$ & $1.78_{-0.30}^{+0.23}$ & & & & & & 3.3 \\
\hline \multirow[t]{2}{*}{ ON231 } & 11-May-1998 & $1.19 / 33$ & $2.58_{-0.06}^{+0.06}$ & $3.09_{-1.38}^{+1.03}$ & $1.58_{-0.44}^{+0.28}$ & & & & & & 4.2 \\
\hline & 11-Jun.-1998 & $0.87 / 31$ & $2.58_{-0.09}^{+0.23}$ & $2.70_{-1.33}^{+0.46}$ & $1.49_{-0.22}^{+0.19}$ & & & & & & 3.4 \\
\hline BLLAC & 31-Oct.-2000 & $1.29 / 146$ & $2.21_{-0.13}^{+0.129}$ & $0.45_{-0.44}^{+0.39}$ & $2.64_{-0.05}^{+0.02}$ & & & & & & 20.2 \\
\hline \multicolumn{12}{|c|}{ FSRQs } \\
\hline $1 \mathrm{ES} 0836+710$ & 27-Мау-1998 & $1.14 / 146$ & $1.08_{-0.23}^{+0.15}$ & $0.38_{-0.37}^{+0.37}$ & $1.34_{-0.02}^{+0.04}$ & & & & & & 26.3 \\
\hline \multirow[t]{5}{*}{ 3C 273} & 18-Jul.-1996 & $1.10 / 229$ & $2.12_{-1.19}^{-0.23}$ & $0.37_{-0.07}^{-0.37}$ & $1.58_{-0.01}^{-0.02}$ & & & & & & 70.1 \\
\hline & 24-Jun.-1998 & $1.22 / 146$ & $1.97_{-0.06}^{-1.09}$ & $0.72_{-0.13}^{+0.11}$ & $1.61_{-0.01}^{+0.01}$ & & & & & & 108.0 \\
\hline & 09-Jan.-2000 & $1.13 / 146$ & $1.90_{-0.05}^{-0.06}$ & $0.84_{-0.16}^{+0.13}$ & $1.63_{-0.01}^{-0.01}$ & & & & & & 116.0 \\
\hline & 13-Jun.-2000 & $0.98 / 146$ & $2.07_{-0.12}^{-0.033}$ & $0.58_{-0.15}^{+0.20}$ & $1.64_{-0.01}^{-0.01}$ & & & & & & 86.7 \\
\hline & 12-Jun.-2001 & $1.06 / 146$ & $2.21_{-0.07}^{+0.08}$ & $0.82_{-0.06}^{+0.11}$ & $1.65_{-0.02}^{+0.02}$ & & & & & & 101.0 \\
\hline PKS 1510-089 & 03-Aug.-1998 & $0.80 / 32$ & $2.51_{-0.30}^{-0.030}$ & $1.41_{-0.25}^{+1.06}$ & $1.32_{-0.10}^{-0.02}$ & & & & & & 5.5 \\
\hline PKS 2126-158 & 24-May-1999 & $0.98 / 146$ & $1.03_{-0.12}^{+0.12}$ & $\begin{array}{r}0.60_{-0.58}^{+6.66} \\
\end{array}$ & $1.78_{-0.11}^{+0.27}$ & & & & & & 10.7 \\
\hline
\end{tabular}

Columns: 1 = object name; $2=$ observation date; $3=$ reduced $\chi^{2}$ and degrees of freedom; $4=$ first photon spectral index; $5=$ energy break in $\mathrm{keV} ; 6=$ second photon spectral index. Columns 3 to 6 refer to fits obtained using the broken power law or the continuously curved parabola model with intrinsic absorption fixed to the Galactic value; $7=$ reduced $\chi^{2}$ and degrees of freedom; $8=$ intrinsic absorption; $9=$ first photon spectral index; $10=$ energy break in $\mathrm{keV} ; 11=$ second photon spectral index. Columns 7 to 11 refer to fits obtained using the broken power law or the continuously curved parabola model with intrinsic absorption free to vary. The intrinsic absorption is in units of $10^{20} \mathrm{~cm}^{-2} ; 12=\mathrm{X}-$ ray unabsorbed flux in the energy range $2-10 \mathrm{keV}$ (in units of $10^{-12} \mathrm{erg} \mathrm{cm}^{-2} \mathrm{~s}^{-1}$ ). 
D. Donato et al.: A BeppoSAX spectral catalog of blazars, Online Material p 9

Table 5. Single power law fits for Spectra in Table 4.

\begin{tabular}{|c|c|c|c|c|}
\hline $\begin{array}{l}\text { Obj. name } \\
\text { (1) }\end{array}$ & $\begin{array}{l}\text { Obs. date } \\
\text { (2) }\end{array}$ & $\begin{array}{c}\chi_{r}^{2} / \text { d.o.f. } \\
\text { (3) }\end{array}$ & $\begin{array}{l}N_{\mathrm{H}} \\
(4)\end{array}$ & $\begin{array}{c}\Gamma \\
(5)\end{array}$ \\
\hline \multicolumn{5}{|c|}{ HBLs } \\
\hline PKS 0548-322 & 20-Feb.-1999 & $1.29 / 95$ & $3.2_{-06}^{+0.8}$ & $2.12_{-0.06}^{+0.07}$ \\
\hline \multirow[t]{2}{*}{ 1ES $1101-232$} & 04-Jan.-1997 & $0.97 / 257$ & $2.4_{-2.0}^{+3.6}$ & $2.01_{-0.06}^{+0.06}$ \\
\hline & 19-Jun.-1998 & $1.19 / 92$ & $3.5_{-1.6}^{+2.0}$ & $2.25_{-0.05}^{+0.06}$ \\
\hline \multirow[t]{16}{*}{ Mkn 421} & 29-Apr.-1997 & $2.28 / 239$ & $2.5_{-2.5}^{+2.6}$ & $2.65_{-0.02}^{+0.05}$ \\
\hline & 30-Apr.-1997 & $2.07 / 239$ & $2.6_{-2.6}^{+2.5}$ & $2.67_{-0.02}^{+0.02}$ \\
\hline & 01-May-1997 & $2.38 / 239$ & $2.4_{-2.4}^{+2.6}$ & $2.63_{-0.02}^{+0.02}$ \\
\hline & 02-May-1997 & $1.81 / 239$ & $2.7_{-02}^{-2.4}$ & $2.68_{-0.04}^{+0.82}$ \\
\hline & 03-May-1997 & $1.62 / 239$ & $2.4_{-0.1}^{+0.2}$ & $2.74_{-0.04}^{+0.84}$ \\
\hline & 04-May-1997 & $1.45 / 239$ & $2.6_{-02}^{+0.1}$ & $2.90_{-0.05}^{+0.04}$ \\
\hline & 05-May-1997 & $1.79 / 239$ & $2.5_{-0.2}^{+0.1}$ & $\begin{array}{l}2.78_{-0.03}^{+0.05} \\
2.04\end{array}$ \\
\hline & 07-May-1997 & $1.14 / 46$ & $1.1_{-0.2}^{+0.2}$ & $2.42_{-0.05}^{+0.03}$ \\
\hline & 21-Apr.-1998 & $7.82 / 155$ & $2.0_{-0.1}^{+0.2}$ & $2.51_{-0.01}^{+0.05}$ \\
\hline & 23-Apr.-1998 & $8.41 / 155$ & $1.9_{-0}^{+0.1}$ & $2.37_{-0.01}^{+0.81}$ \\
\hline & 22-Jun.-1998 & $4.32 / 155$ & $2.1_{-0.2}^{+0.1}$ & $2.43_{-0.01}^{+0.01}$ \\
\hline & 04-May-1999 & $5.93 / 155$ & $2.2_{-0.1}^{+0.2}$ & $2.76_{-0.01}^{+0.81}$ \\
\hline & 26-Apr.-2000 & $2.53 / 155$ & $1.5^{+0.1}$ & $2.02^{+0.81}$ \\
\hline & 28-Apr--2000 & $\begin{array}{l}2.53 / 153 \\
7.00 / 155\end{array}$ & $\begin{array}{l}1.5-0.1 \\
1.4^{+0.1}\end{array}$ & $\begin{array}{l}2.02-0.01 \\
2.00^{+0.01}\end{array}$ \\
\hline & 30-Apr.-2000 & $3.95 / 155$ & $\begin{array}{l}1.4-0.1 \\
1.3^{+0.1}\end{array}$ & $\begin{array}{l}2.00-0.01 \\
2.01^{+0.01}\end{array}$ \\
\hline & 09-May-2000 & $3.73 / 155$ & $1.3_{-0.1}^{+0.1}$ & $2.09_{-0.01}^{+0.81}$ \\
\hline RX J1117.1+2014 & 13-Dec.-1999 & $1.36 / 85$ & 3. & $2.86_{-0.06}^{+0.01}$ \\
\hline $1 \mathrm{ES} 1218+304$ & 12-Jul.-1999 & $0.94 / 95$ & $2.9_{-0.5}^{+0.4}$ & $\begin{array}{l}-0.06 \\
2.51^{+0.05}\end{array}$ \\
\hline \multirow{2}{*}{ 1RXS J141756.8+25 } & 13-Jul.-2000 & $1.12 / 94$ & $2.8_{-0.7}^{+0.4}$ & $2.23_{-0.07}^{+0.88}$ \\
\hline & 23-Jul.-2000 & $1.16 / 39$ & $\begin{array}{l}2.0-0.7 \\
2.1_{-1}^{+1.3}\end{array}$ & $2.22_{-0.15}^{+0.07}$ \\
\hline 1ES $1517+656$ & 05-Mar.-1997 & $1.76 / 40$ & $\begin{array}{l}2.1-1.2 \\
16.3_{-42}^{+5.8}\end{array}$ & $\begin{array}{l}2.49_{-0.13}^{+0.15} \\
2.13\end{array}$ \\
\hline 1ES $1553+113$ & 05-Feb.-1998 & $1.13 / 68$ & $6.9_{-44}^{+5.3^{2}}$ & $2.33_{-0.17}^{+0.13}$ \\
\hline \multirow[t]{11}{*}{ Mkn 501} & 07-Apr.-1997 & $1.15 / 236$ & $1.3_{-0.1}^{-4.4}$ & $1.90_{-0.02}^{+0.01}$ \\
\hline & 11-Apr.-1997 & $1.04 / 233$ & $0.9_{-0.1}^{+0.1}$ & $\begin{array}{l}1.90_{-0.02} \\
1.80_{-0.02}^{+0.02}\end{array}$ \\
\hline & 16-Apr.-1997 & $1.41 / 236$ & $0.9_{-0.1}^{+0.1}$ & $\begin{array}{l}1.00-0.02 \\
1.59^{+0.01}\end{array}$ \\
\hline & 28-Apr.-1998 & $\begin{array}{l}1.41 / 250 \\
1.49 / 147\end{array}$ & $\begin{array}{l}0.9-0.1 \\
1.0^{+0.1}\end{array}$ & $\begin{array}{l}1.59-0.01 \\
1.83^{+0.02}\end{array}$ \\
\hline & 29-Apr.-1998 & $1.37 / 142$ & $\begin{array}{l}1.0 .2 \\
1.1^{+0.1}\end{array}$ & $\begin{array}{l}1.03-0.02 \\
1.82^{+0.02}\end{array}$ \\
\hline & 01-May-1998 & $1.74 / 144$ & $1.4^{+0.2}$ & $\begin{array}{l}1.02^{-0.02} \\
1.98^{+0.03}\end{array}$ \\
\hline & 20-Jun.-1998 & $1.07 / 147$ & $\begin{array}{l}1.4-0.1 \\
1.2_{-0.2}^{+0.2}\end{array}$ & $\begin{array}{l}1.90-0.02 \\
2.03_{-0.03}^{+0.03}\end{array}$ \\
\hline & 29-Jun.-1998 & $1.25 / 147$ & $\begin{array}{l}1 .-0.2 \\
1.4_{-02}^{+0.1}\end{array}$ & $1.96_{-0.03}^{+0.03}$ \\
\hline & 16-Jul.-1998 & $1.69 / 147$ & $1.9_{-0.2}^{+0.3}$ & $2.07_{-0.03}^{+0.03}$ \\
\hline & 25-Jul.-1998 & $1.93 / 147$ & $1.5_{-02}^{+0.1}$ & $2.06_{-0.03}^{+0.032}$ \\
\hline & 10-Jun.-1999 & $1.78 / 147$ & $1.3_{-0.1}^{-0.2}$ & $2.41_{-0.02}^{+0.01}$ \\
\hline $\mathrm{H} 1722+119$ & 24-Aug.-2001 & $1.29 / 147$ & $12.6_{-13}^{+1.13}$ & $2.56_{-0.03}^{+0.02}$ \\
\hline \multirow[t]{3}{*}{ 1ES $1959+650$} & 04-May-1997 & $1.02 / 45$ & $13.3_{-6.2}^{+7.5}$ & $2.70_{-0.12}^{+0.03}$ \\
\hline & 23-Sep.-2001 & $1.35 / 147$ & $12.5^{+6.2 .1}$ & $2.51_{-0.05}^{+0.05}$ \\
\hline & 28-Sep.-2001 & $1.28 / 144$ & $20.9^{+0.9}$ & $2.33_{-0.02}^{+0.05}$ \\
\hline PKS 2005-489 & 01-Nov.-1998 & $1.95 / 145$ & & $2.19^{+0.01}$ \\
\hline \multirow[t]{3}{*}{ PKS 2155-304 } & 20-Nov.-1996 & $2.18 / 231$ & $1.3_{-0.1}^{+0.1}$ & $2.63_{-0.01}^{+0.81}$ \\
\hline & 22-Nov.-1997 & $5.70 / 147$ & $2.0^{+0.1}$ & $2.70^{+0.01}$ \\
\hline & 04-Nov.-1999 & $2.22 / 147$ & $0.9_{-02}^{+0.1}$ & $2.80_{-0.01}^{+0.81}$ \\
\hline 1ES $2344+514$ & 03-Dec.-1999 & $1.41 / 92$ & $11.8_{-36}^{+4.1}$ & $2.33_{-0.05}^{+0.01}$ \\
\hline H2356-309 & 21-Jun.-1998 & $1.33 / 147$ & $\begin{array}{l}2.1_{-0.4}^{+0.4} \\
0\end{array}$ & $\begin{array}{l}2.09_{-0.04}^{+0.05} \\
2.03\end{array}$ \\
\hline \multicolumn{5}{|c|}{ LBLs } \\
\hline $3 \mathrm{C} 66 \mathrm{~A}$ & 31-Jan.-1999 & $1.26 / 35$ & & $2.26_{-0.17}^{+0.18}$ \\
\hline \multirow[t]{3}{*}{ S50716+71 } & 14-Nov.-1996 & $1.18 / 49$ & $0.1_{-0.1}^{+0.1}$ & $2.06_{-0.13}^{+0.17}$ \\
\hline & 07-Nov.-1998 & $2.06 / 35$ & & $2.21^{+0.13}$ \\
\hline & 30 -Oct.-2000 & $1.55 / 34$ & & $2.05^{+0.13}$ \\
\hline \multirow[t]{2}{*}{ ON231 } & 11-May-1998 & $2.86 / 35$ & & $2.50_{-0.14}^{+0.12}$ \\
\hline & 11-Jun.-1998 & $3.04 / 34$ & & $2.36_{-0.14}^{+0.14}$ \\
\hline BLLAC & 31-Oct.-2000 & $1.28 / 147$ & $10.1_{-2.3}^{+2.3}$ & $2.65_{-0.05}^{+0.06}$ \\
\hline \multicolumn{5}{|c|}{ FSRQs } \\
\hline 1ES $0836+710$ & 27-May-1998 & $1.13 / 147$ & $78.0_{-352}^{+54.9}$ & $1.34_{-0.04}^{+0.04}$ \\
\hline $3 \mathrm{C} 273$ & 18-Jul.-1996 & $1.14 / 231$ & & $1.58^{+0.001}$ \\
\hline & 24-Jun.-1998 & $2.22 / 148$ & & $1.65^{+0.001}$ \\
\hline & 09-Jan.-2000 & $2.10 / 148$ & & $1.67^{-0.01}$ \\
\hline & 13-Jun.-2000 & $\begin{array}{l}.10 / 140 \\
1.79 / 148\end{array}$ & & $1.68_{-0.01}^{+0.81}$ \\
\hline & 12-Jun.-2001 & $3.08 / 148$ & & $1.74_{-0.01}^{+0.01}$ \\
\hline PKS 1510-089 & 03-Aug.-1998 & $1.37 / 35$ & & $1.37_{-0.10}^{+0.81}$ \\
\hline PKS 2126-158 & 24-May-1999 & $1.06 / 147$ & $294.1_{-86.5}^{+110.7}$ & $1.62_{-0.04}^{+0.04}$ \\
\hline
\end{tabular}

Columns: 1 = object name; $2=$ observation date; $3=$ reduced $\chi^{2}$ and degrees of freedom; $4=$ free intrinsic absorption $\left(\right.$ in $u n i t s ~ o f ~ 10^{20} \mathrm{~cm}^{-2}$ ); $5=$ photon spectral index for the single power law model. 